Aus der Abteilung Anaesthesiologie

(Prof. Dr. med. M. Quintel)

im Zentrum Anaesthesiologie, Rettungs- und Intensivmedizin

der Medizinischen Fakultät der Universität Göttingen

\title{
Vergleich der laryngoskopischen Intubation versus Intubation über die Intubationslarynxmaske an Patienten
}

\author{
Inaugural - Dissertation \\ zur Erlangung des Doktorgrades \\ der Medizinischen Fakultät \\ der Georg-August-Universität zu Göttingen
}

vorgelegt von

Birgit Mundt

aus

Holzminden

Göttingen 2012 
De k a n:

I. Berichterstatter:

II. Berichterstatter/in:

III. Berichterstatterlin:

Tag der mündlichen Prüfung:
Prof. Dr. med. M.P. Schön

PD Dr. med. A. Timmermann 


\section{INHALTSVERZEICHNIS}

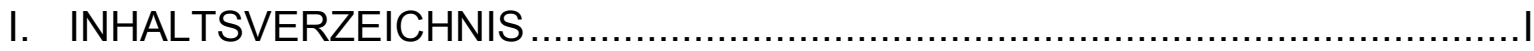

II. ABKÜRZUNGEN, ABBILDUNGEN, TABELLEN UND

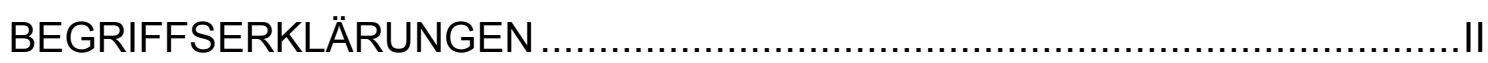

II.1 Liste der verwendeten Abkürzungen .............................................

II.2 Abbildungsverzeichnis ..................................................................

II.3 Tabellenverzeichnis ............................................................... IV

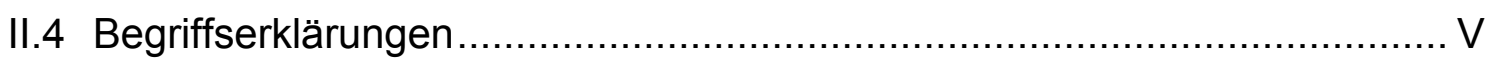

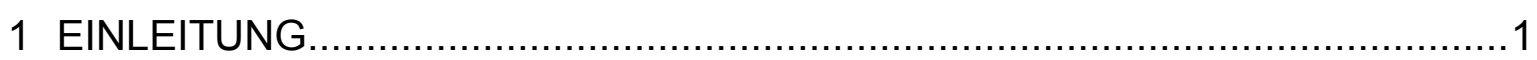

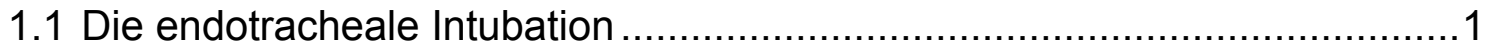

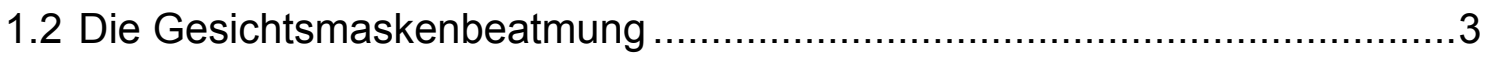

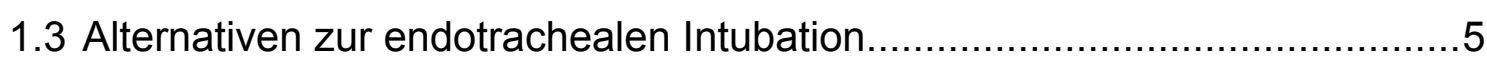

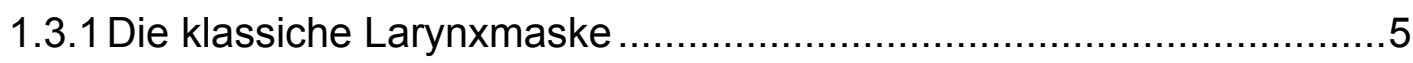

1.3.2 Die Intubations-Larynxmaske ............................................. 6

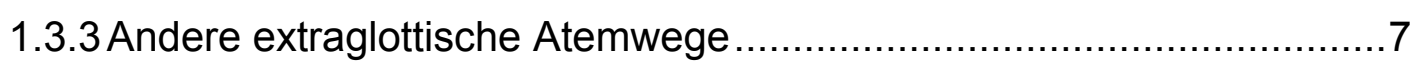

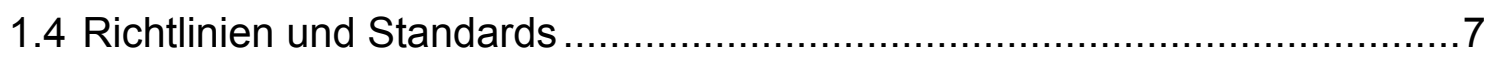

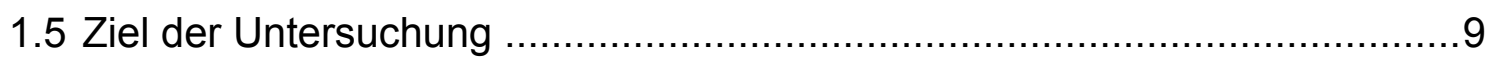

2 MATERIAL UND METHODEN ............................................................ 10

2.1 Rekrutierung der Probanden........................................................ 10

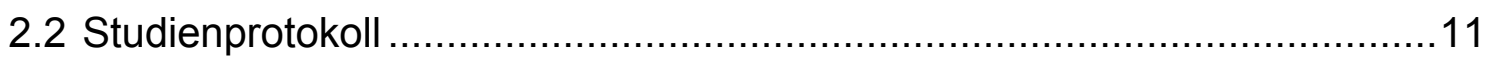

2.2.1 Trainingsphase ...................................................................... 11

2.2.2 Rekrutierung der Patienten............................................... 17

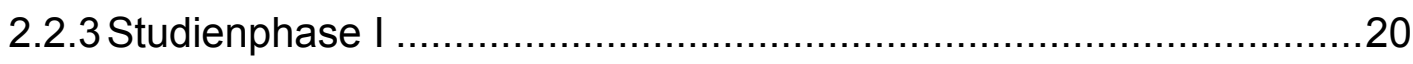

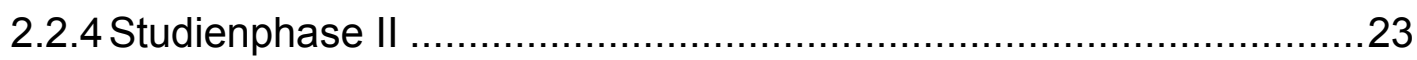

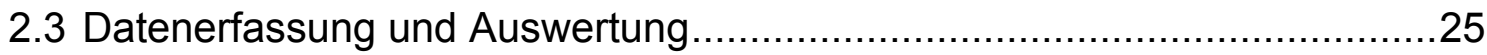

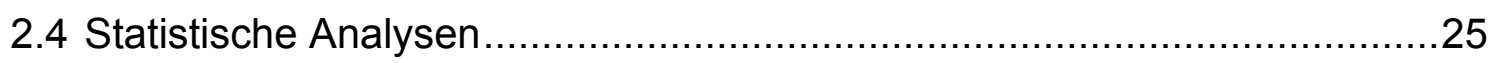

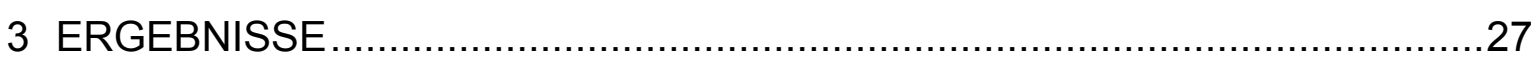

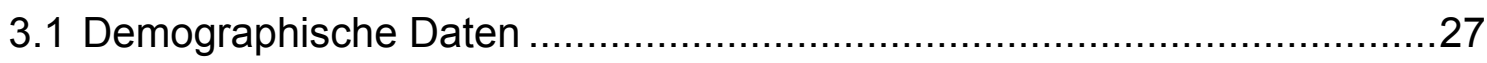

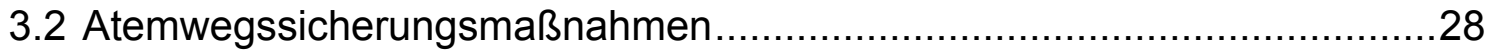

3.2.1 Ventilationsmaßnahmen ..................................................... 28

3.2.2 Intubationsmaßnahmen....................................................... 30

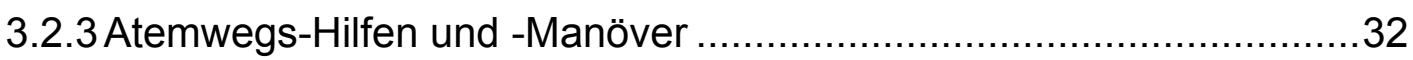

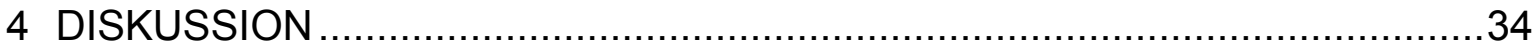

5 ZUSAMMENFASSUNG UND SCHLUSSFOLGERUNG ...........................41

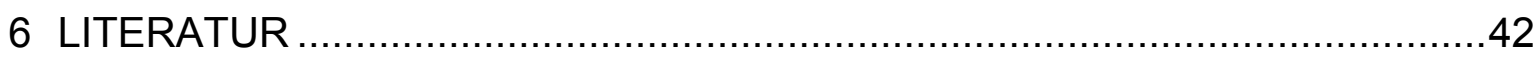




\section{Abkürzungen, Abbildungen, Tabellen und Begriffserklärungen}

\section{II.1 Liste der verwendeten Abkürzungen}

ASA

BM-V

CRP

ERC

ETI

ETT

$\mathrm{FiO}_{2}$

ILCOR

ILMA

ILMA-TI

ILMA-V

LG-TI

LMA

NIV

NRettDG

OP

SGA
American Society of Anaesthesiology

Beutel-Gesichtsmaskenbeatmung

kardiopulmonale Reanimation

European Resuscitation Council

endotracheale Intubation

Endotrachealtubus

inspiratorische Sauerstoffkonzentration

International Liaison Committee on Resuscitation

Intubations-Larynxmaske

Intubation via Intubations-Larynxmaske

Ventilation via Intubations-Larynxmaske

laryngoskopisch durchgeführte endotracheale Intubation

Larynxmaske

nichtinvasive Beatmung

Niedersächsisches Rettungsdienstgesetz

Operationssaal

supraglottischer Atemweg 


\section{II.2 Abbildungsverzeichnis}

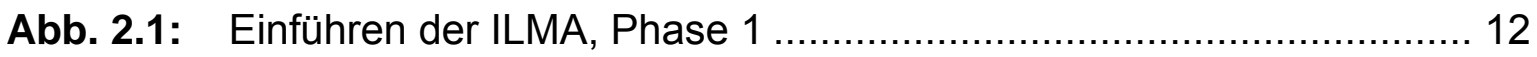

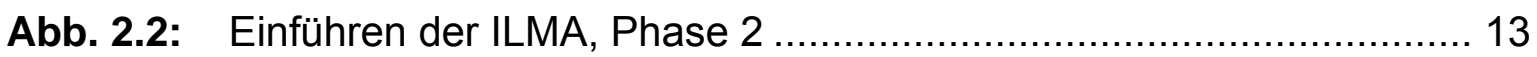

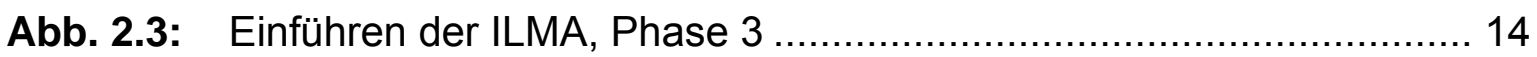

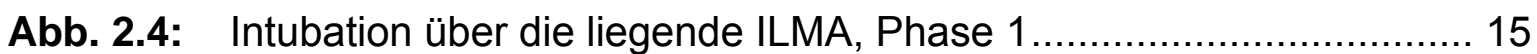

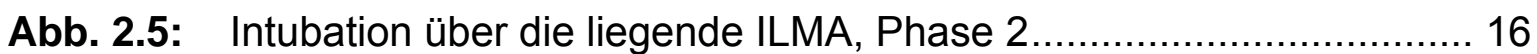

Abb. 2.6: Graphische Darstellung des Versuchsablaufs.............................. 24

Abb. 3.1: Kaplan-Meier-Analyse, Ventilation …....................................... 30

Abb. 2.6: Kaplan-Meier-Analyse, Intubation ............................................ 32 


\section{II.3 Tabellenverzeichnis}

Tab. 2.1: Patientenzahlen bezogen auf die operativen Disziplinen.

Tab. 3.1: Demographische und deskriptive Daten

Tab. 3.2: Erfolgsraten und benötigte Zeit zur Ventilation .28

Tab. 3.3: Erfolgsraten und benötigte Zeit zur Intubation .31

Tab. 3.4: Verwendete Atemwegs-Hilfen und -Manöver .33 


\section{II.4 Begriffserklärungen}

Aspiration:

Beutel-

Gesichtsmasken-

beatmung:

Body Mass Index:

BURP-Manöver:

Chandi-Manöver:

Combitubus:
Ansaugen, Einatmen von Luft oder Flüssigkeit. Eindringen flüssiger oder fester Stoffe in die Atemwege (Mageninhalt, Blut, Fremdkörper).

Beatmung mit Hilfe einer Beatmungsmaske, an die ein Reservoir-Beutel angeschlossen ist, und durch den die Beatmung mittels Kompression des Beutels möglich ist.

Messzahl zur Bewertung des Körpergewichts. Körpermasse in Kilogramm bezogen auf die Körpergröße im Quadrat. Adipositas-Klassifikation der WHO.

Maßnahme, um die konventionelle endotracheale Intubation zu erleichtern. Die Abkürzung steht für: backward, upward, rightward pressure. Dabei wird ein nach dorsal, kranial und rechts gerichteter Druck von außen auf den Schildknorpel ausgeübt.

Maßnahme, um die endotracheale Intubation über die ILMA zu erleichtern. Der Handgriff der ILMA wird nach dem Einführen nach dorsal bewegt, so dass sich das distale Ende der ILMA nach ventral verschiebt, und der Pharynx um einige Millimeter angehoben wird. Dadurch wird eine optimale Position der ILMA zur trachealen Achse erreicht, und die ILMA-Intubation erleichtert.

Der Combitubus kann als Doppellumentubus entweder im Ösophagus oder in der Trachea positioniert werden. Das als "Ösophageal" bezeichnete Lumen hat ein distal verschlossenes Ende sowie Perforationen in der Rachenebene (Pharynxebene). Das als "tracheal“ bezeichnete Lumen zeigt ein distal offenes Ende entsprechend einem Endotrachealtubus. Die Lumina sind durch eine Membran voneinander getrennt, am proximalen Ende sind die beiden Lumina mittels eines kurzen Tubus mit einem Konnektor verbunden. Oberhalb 
der Perforation ist der Oropharyngeal-Ballon angebracht, zwei Ringmarken am oberen Ende geben die Einführungstiefe an. Am distalen Ende dient ein herkömmlicher Ballon zur Abdichtung von Speiseröhre oder Luftröhre.

Cuff:

Aufblasbare, distal am Tubus angebrachte Manschette eines Endotrachealtubus, der über einen teilweise in die Tubuswand eingearbeiteten Füllschlauch aufgebläht werden kann. Somit ist sicher gestellt, dass das Bronchialsystem gegen Sekret und feste Stoffe abgedichtet ist.

einseitige

Intubation:

endotracheale

Intubation:

Endotrachealtubus: Beatmungsschlauch, der mit dem distalen Lumen in der Trachea zu liegen kommt, und über den die Beatmung eines Patienten ermöglicht wird.

Epiglottisheber: Kunststofflippe, die an der ILMA befestigt ist und beim Einführen des Endotrachealtubus angehoben wird, und damit die Epiglottis anhebt und so den Weg auf den Kehlkopf frei gibt.

Güdel-Tubus: $\quad$ Gekrümmter oropharyngealer Tubus zum kurzfristigen Freihalten der oberen Atemwege; verhindert das Zurückweichen der Zunge und die Verlegung der oberen Luftwege.

Intubations-

Larynxmaske:

Kapnographie:

Kreissystem:
Kehlkopfmaske, durch die ein Endotrachealtubus eingeführt werden kann.

Graphische Darstellung des $\mathrm{CO}_{2}$-Gehaltes der Atemluft im Atemzyklus.

Beim Narkosesystem wird das ausgeatmete Gasvolumen (teilweise oder vollständig) wieder zum Patienten 
zurückgeführt. Das hierfür notwendige Schlauchsystem wird als Kreissystem bezeichnet.

Laryngoskop:

Instrument, mit dem die Sicht auf den Larynx ermöglicht wird. Konventionelle Laryngoskope bestehen aus einem Griff und einem Spatel, der in unterschiedlichen Formen und Größen angewendet werden kann.

Larynxmaske:

Die Kehlkopfmaske besteht aus einem aufblasbaren, blockbaren, ovalen Silikonkörper, an dem ein flexibler Schlauch tubusartig angebracht ist, und mit einem 15-mm-Konnektor versehen ist. Der Silikonkörper hat die Aufgabe, den Raum um und hinter dem Kehlkopf abzudichten. An der Innenseite der Maske befindet sich eine Öffnung, die von zwei dünnen Silikonstreben durchbrochen wird. Diese Öffnung soll im Idealfall der Epiglottis gegenüber liegen. Die Streben haben die Funktion, die Epiglottis an einem Einfallen in die Öffnung zu hindern und so den Luftweg frei zu halten. Die LMA wird ohne direkte Sicht auf den Kehlkopf (blind) eingeführt.

Larynxtubus: Der Larynxtubus besteht aus einem Tubus mit einem pharyngealen und einem ösophagealen Cuff aus Silikon. Diese Cuffmanschetten korrespondieren miteinander, und sind durch ein Schlauchsystem zu befüllen. Das Einführen erfolgt ohne direkte Sicht (blind), bis die am Tubus angebrachte Strichmarkierung auf Höhe der oberen Zahnreihe zu liegen kommt. Der pharyngeale und ösophageale Cuff wird mit Luft gefüllt. Durch die spezielle Cuffzuleitung wird zuerst der pharyngeale Cuff gefüllt. Dadurch kommt es zu einer Anpassung an die Strukturen des Pharynx und zu einer Stabilisierung des Tubus. Danach erfolgt automatisch die Füllung des ösophagealen Cuffs. Der ösophageale Cuff verschließt den Ösophaguseingang, der pharyngeale den Mund- und Rachenraum. Zwischen den Cuffs endet das Lumen in Höhe des Kehlkopfes, so dass durch die Abdichtung der Manschetten nach oben und unten die durch das Beatmungsgerät eingebrachte Luft in die Lunge strömen 
kann.

Lungencompliance: Compliance ist ein Maß für die Dehnbarkeit von Körperstrukturen, hier des Lungengewebes. Sie wird zur Beschreibung und Quantifizierung der elastischen Eigenschaften der betrachteten Gewebe gebraucht. Die Compliance gibt an, wie viel Volumen (Gas oder Flüssigkeit) in eine umwandete Struktur aufgenommen werden kann, bis der Druck um eine Druckeinheit ansteigt. Die Maßeinheit ist I/kPa.

MacIntosh-Spatel Das Intubationslaryngoskop besteht aus einem Griff und einem sog. Spatel, dieser Spatel kann vom Handgriff gelöst werden. Spatel stehen in unterschiedlichen Größen und Formen zur Verfügen, sodass eine bedarfsadaptierte Anwendung möglich ist. Der MacIntosh-Spatel ist eine Variante eines Intubationsspatels. Er ist leicht gebogen, und je nach Größe zwischen 76 mm (Größe 0) und 176 mm (Größe 5) lang. Bei Erwachsenen werden häufig die Größen 3 und 4 verwendet.

MallampatiKlassifikation:

Narkoseeinleitung: Die Narkoseeinleitung ist die medikamentöse Induktion einer Allgemeinanästhesie.

Nichtinvasive

Beatmung:

Die Mallampati-Klassifikation dient zur Abschätzung des Schwierigkeitsgrades einer endotrachealen Intubation vor der Einleitung einer Narkose. Zur Beurteilung wird der aufrecht sitzende Patient aufgefordert, bei neutraler Position des Kopfes die Zunge so weit wie möglich herauszustrecken. Dabei wird die Sichtbarkeit der oropharyngealen Strukturen beurteilt. Zwischen der Einsehbarkeit der pharyngealen Strukturen nach dem Mallampati- Score und der Leichtigkeit der endotrachealen Intubation besteht eine positive Korrelation. Die Einteilung erfolgt in vier Grade.

Unter dem Begriff nichtinvasive Beatmung (noninvasive Ventilation) werden alle Beatmungsformen zusammen gefasst, bei denen keine endotracheale Intubation durchgeführt wird. Die Beatmung erfolgt häufig mit Hilfe 
einer Maske, die an ein Beatmungsgerät angeschlossen werden kann.

Präoxygenierung: Als Präoxygenierung bezeichnet man eine prophylaktische Anreicherung der Inspirationsluft mit Sauerstoff vor einer induzierten Apnoe, zum Beispiel im Rahmen einer Narkoseeinleitung. Durch die Zufuhr von Sauerstoff wird der Sauerstoffspeicher der Lunge (entspricht der funktionellen Residualkapazität) mit Sauerstoff gefüllt, wobei der Stickstoff der Atemluft "ausgewaschen" wird, was einer Denitrogenisierung entspricht. Dadurch wird die Apnoetoleranz des Patienten erhöht.

Protrusion des

Unterkiefers:

Supraglottischer

Atemweg:

Tidalvolumen:

Up-and-down-

Manöver:

Zweihand-

Maskenbeatmung:
Das Vorschieben des Unterkiefers vor die obere Zahnreihe.

Atemwegshilfen, bei denen die Glottisebene nicht passiert wird, so dass die Atemwegshilfe oberhalb des Glottisbereiches zu liegen kommt. Supraglottische Atemwegshilfen werden auch als extraglottische Atemwegshilfen bezeichnet.

Atemzugvolumen

Das Zurück- und Wieder-Vorschieben der geblockten ILMA zur Verbesserung der Intubationsbedingungen. Dieses Manöver kommt dann zur Anwendung, wenn die Epiglottis nach kaudal verlagert wurde und die Stimmritze verlegt.

Form der Beutel-Gesichtsmaskenbeatmung, bei der die Beatmungsmaske mit beiden Händen fixiert wird; dies erfordert die Anwesenheit eines zweiten Helfers, um die Beatmung durchzuführen. 


\section{$1 \quad$ Einleitung}

In der Notfallmedizin ist die Sicherung und Freihaltung der Atemwege ein elementarer Bestandteil der Rettungsmaßnahmen, da ohne freie Atemwege und adäquaten Gasaustausch alle anderen Therapiemaßnahmen vergeblich bleiben (Dirks 2001). In der präklinischen Behandlung ist das Atemwegsmanagement ein darüber hinaus besonders zeitkritischer Faktor, der für die Prognose des Notfallpatienten von entscheidender Bedeutung ist.

\subsection{Die endotracheale Intubation}

„Die endotracheale Intubation (ETI) ist der Goldstandard der Atemwegssicherung" (Nolan et al. 2005, S.54). Voraussetzung hierfür ist die fundierte Ausbildung des notfallmedizinischen Personals, sowie die routinemäßige Ausübung dieser Intervention (Nolan et al. 2005; Nolan et al. 2010).

Die Vorteile der ETI bei respiratorisch insuffizienten Patienten, gegenüber anderen Maßnahmen der Atemwegssicherung sind offensichtlich. Die ETI gewährleistet ein Offenhalten der Atemwege. Die Beatmung eines Patienten kann mit Hilfe eines Respirators erfolgen und die Oxygenierung kann durch Applikation einer inspiratorischen Sauerstoffkonzentration $\left(\mathrm{FiO}_{2}\right)$ von bis zu 1,0 gewährleistet werden. Bei Bedarf können endotracheal Medikamente verabreicht werden. Nach erfolgter Platzierung des Endotrachealtubus (ETT) besteht Aspirationsschutz (Timmermann 2009).

Die Vorteile gegenüber anderen Techniken zur Atemwegssicherung, wie beispielsweise der Beutel-Gesichtsmaskenbeatmung (BM-V), der nichtinvasiven Beatmung (NIV) oder der Verwendung eines supraglottischen Atemweges (SGA), sind vielfältig. Dazu zählt im Bedarfsfall die Beatmung mit hohen inspiratorischen Atemwegsdrücken, wie es zum Beispiel beim schweren Lungentrauma notwendig sein kann. Der Aufbau hoher positiver inspiratorischer Drücke ist via Endotrachealtubus problemlos möglich. Tracheobronchiales Sekret kann effektiv abgesaugt werden. Durch blockbare Endotrachealtuben (ETT) ist das Risiko einer Insufflation von Luft in den Verdauungstrakt minimiert. Im Gegensatz dazu wird bei Verwendung der BM- $\mathrm{V}$, der nichtinvasiven Beatmung oder bei einem fehlplazierten 
SGA der Atemwegsdruck auch auf den Ösophagus ausgeübt. Wird hierbei der Verschlussdruck des unteren Ösophagussphinkters überschritten, kommt es zur Insufflation von Luft in den Magen. Die Folgen sind fatal, denn mit steigendem Luftvolumen, nimmt der intragastrale Druck zu, und es kommt zu einer Verlagerung des Zwerchfells nach kranial. Die Lungencompliance verringert sich in der Folge, so dass die erforderlichen Beatmungsdrücke weiter steigen, und immer mehr Luft in den Magen insuffliert wird. Es beginnt ein Circulus vitiosus mit zunehmend verminderter Lungenventilation (Wenzel et al. 1998; Timmermann 2009).

Die erfolgreiche endotracheale Intubation (ETI) gelingt in bis zu 98\% der Fälle bei ärztlichem und nichtärztlichem notfallmedizinischem Personal. Diese Angaben beruhen auf Selbsteinschätzungen der Anwender (Adnet et al. 1998; Bulger et al. 2002; Slagt et al. 2004). Die Angaben des Intubationserfolges wurden jedoch nicht von unabhängigen Beobachtern erhoben, und teilweise unvollständig dokumentiert. Der Bericht einer erfolglosen Intubation mit möglicherweise deletärem Ausgang impliziert unter Umständen eine befangene Dokumentation (Nolan 2001; Timmermann et al. 2006).

Wird hingegen der Intubationserfolg durch unabhängige Beobachter bewertet, so wird die Rate unerkannter ösophagealer Intubationen mit $6-25 \%$ bei Ankunft im Schock-Op angegeben, wenn US-Paramedics (Rettungsassistenten) die endotracheale Intubation ohne Kontrolle des endtidalen $\mathrm{CO}_{2}$ durchgeführt hatten (Katz und Falk 2001; Jemmett et al. 2003; Jones et al. 2004; Silvestri et al. 2005). Leider ist dieses Phänomen auch im deutschen Rettungsdienst beschrieben worden. In einer 5-Jahres-Studie wurde die Inzidenz an einseitigen Intubationen in über 10\% angegeben und weitere $7 \%$ der Patienten waren unerkannt fehlintubiert (Timmermann et al. 2007a).

Daher wird die ETI als Maßnahme zur Atemwegssicherung in der Notfallmedizin zunehmend kritisiert. Trotz der potentiellen Vorteile der ETI konnte in einigen Untersuchungen kein Nutzen gegenüber der Gesichtsmaskenbeatmung gezeigt werden (Gausche et al. 2000; Wang et al. 2004). Dies gilt vor allem für medizinisches Personal, das die ETI nicht regelmäßig durchführt. Um die Technik der endotrachealen Intubation $\mathrm{zu}$ erlernen, werden mindestens 50 bis 60 Intubationsdurchgänge als notwendig erachtet, die elektiv und ohne Prädiktoren erschwerter Atemwegsverhälnisse unter den idealen Bedingungen im Operationssaal 
durchgeführt werden sollten. Dabei ist die individuelle Lernkurve der Intubation an Patienten jedoch sehr heterogen (Konrad et al. 1998; Mulcaster et al. 2003). Die Sicherung der Atemwege in Notfallsituationen erfolgt durch unterschiedliche medizinische Berufsgruppen. Das Erlernen der endotrachealen Intubation ist für Personen, die nicht anästhesiologisch tätig sind, häufig allein aus Zeitgründen, nicht ausreichend möglich. Weiterhin führt der Einsatz moderner Methoden zur Sicherung der Atemwege, wie beispielsweise die Larynxmaske (LMA), oder die Anwendung regionalanästhesiologischer Verfahren, zu einer Abnahme der Zahl der endotrachealen Intubationen im Routinebetrieb. So ist es nicht verwunderlich, dass in Deutschland nur ca. 1/3 der nicht anästhesiologischen Notärzte in einer anonymen Umfrage angaben, mehr als $100 \mathrm{ETI}$ durchgeführt zu haben (Timmermann et al. 2007a). Ein Fünftel der Notärzte haben die ETI nur selten durchgeführt (weniger als 20 Anwendungen). Hinzu kommt, dass die ETI im Rettungsdienst als deutlich schwieriger eingestuft wird (Timmermann et al. 2006). Somit muss gerade bei diesen Notärzten davon ausgegangen werden, dass jede ETI unter präklinischen Bedingungen eine extrem schwierige Situation darstellt.

Prinzipiell stehen dem notfallmedizinischem Personal die nachfolgenden Möglichkeiten zur Ventilation und Oxygenierung der respiratorisch insuffizienten Notfallpatienten zur Verfügung.

\subsection{Die Gesichtsmaskenbeatmung}

Die Beutel-Gesichtsmaskenbeatmung (BM-V) ist die primäre Beatmungsmöglichkeit für professionelle Helfer während Notfallsituationen. Die suffiziente Durchführung ist jedoch technisch schwierig, und gelingt unerfahrenen Personen nicht immer ausreichend, wie Doerges und Mitarbeiter in zwei Studien an Übungsphantomen zeigen konnten (Doerges et al. 1999a; Doerges et al. 1999b). Auch bei der Anwendung der BM-V durch versierte Anästhesisten ermittelten Langeron und Mitarbeiter eine Inzidenz der schwierigen Maskenbeatmung von 5\%, selbst unter optimalen Bedingungen im Operations-Saal (Langeron et al. 2000). Daten zur Inzidenz der schwierigen Maskenbeatmung in präklinischen Situationen liegen derzeit nicht vor.

Bei längerfristiger Anwendung der BM-V nimmt das Aspirationsrisiko zu (Doerges und Wenzel 2001) und steigt während der kardiopulmonalen Reanimation 
(CRP) noch deutlich an. Die Ursachen für ein erhöhtes Aspirationsrisiko sind in einem Nachlassen der Schutzreflexe sowie in einer Reduzierung des Verschlussdrucks des unteren Ösophagusspinkters zu sehen. Bowman et al. konnten im Tierversuch als auch beim Menschen zeigen, dass dieser Verschlussdruck von $20 \mathrm{cmH}_{2} \mathrm{O}$ auf minimal $5 \mathrm{cmH}_{2} \mathrm{O}$ abfallen kann (Bowman et al. 1995; Gabrielli et al. 2005). Dabei beeinflussen sich Ventilations- und Oxygenationsbedingungen gegenseitig negativ. Da die Insufflation von Luft in den Magen bei einem Verschlussdruck von $5 \mathrm{cmH}_{2} \mathrm{O}$ nahezu unvermeidbar ist (Wenzel et al. 1998), resultiert hieraus ein erhöhter intragastraler Druck (Spence et al. 1967), der die Zwerchfellbeweglichkeit einschränkt und die Lungencompliance reduziert. Die verminderte Lungencompliance bewirkt jedoch, dass die notwendigen Atemwegsdrücke steigen, um ein bestimmtes Tidalvolumen zu erreichen, was wiederum mit einer vermehrten Insufflation von Luft in den Magen verbunden ist (Ruben et al. 1961; Wenzel et al. 1998). Dies kann zu einer erhöhten Komplikationsrate von Aspiration und Pneumonien mit möglicher Todesfolge führen (Morton und Wylie 1951; Lawes und Baskett 1987). Thierbach und Mitarbeiter ermittelten die Inzidenz der Aspiration aller notfallmedizinischen Patienten in Deutschland mit $5 \%$, als die häufigste Komplikation (Thierbach et al. 2004). Eine Reduzierung des Tidalvolumens während der BM-V unter CRP wurde aus diesem Grund von Autoren und Fachgesellschaften empfohlen, wobei die Einhaltung des Atemminutenvolumens beachtet werden muss (Wenzel et al. 1999; Doerges et al. 1999a; Doerges et al. 1999b; Doerges et al. 2000; Doerges 2005).

Da auch die Maskenbeatmung zur sicheren Beherrschung einer regelmäßigen Übung bedarf, und in Anbetracht der erheblichen Probleme, die im Verlauf der Maskenbeatmung auftreten können, wurden in den vergangenen Jahren intensiv Alternativen zur BM-V und zur laryngoskopisch durchgeführten endotrachealen Intubation (LG-TI) untersucht, deren wichtigste Ergebnisse im Folgenden kurz dargestellt werden. 


\subsection{Alternativen zur endotrachealen Intubation}

\subsubsection{Die klassiche Larynxmaske}

Die klassische Larynxmaske (LMA) ist die bekannteste supraglottische Atemwegshilfe. Seit 1983 ist die LMA in den Routinebetrieb sowie in die Notfallmedizin eingeführt und hat seitdem weltweite Verbreitung gefunden (Brain 1983; Sasada und Gabbott 1994; Martin et al. 1999). Die Larynxmaske wird ohne laryngoskopische Hilfe oral eingeführt, in den Hypopharynx vorgeschoben und schließlich geblockt. Die Cuffmanschette legt sich der Rachenhinterwand an, wobei sich die Spitze der LMA im oberen Ösophagussphinkter befindet. Die ventral liegende Beatmungsöffnung weist auf den Kehlkopfeingang. Ein vollständiger Aspirationsschutz bei Anwendung der LMA ist nicht gewährleistet, jedoch ist die Aspiration unter elektiven Bedingungen ausgesprochen unwahrscheinlich. Brimacombe gibt die Inzidenz einer Aspiration laut Metaanalyse mit $0,012 \%$ an (Brimacombe 2005). Die klassische LMA ist für Patienten aller Altersstufen und Gewichtsklassen erhältlich.

Die LMA bietet im Vergleich zur ETI im präklinischen Atemwegsmanagement Vorteile, sowohl für Experten, als auch für unerfahrene Anwender. So konnte in mehreren Studien gezeigt werden, dass Rettungsdienstpersonal mit nur geringer Erfahrung im Atemwegsmanagement die LMA schneller und erfolgreicher platzieren konnte, als den Endotrachealtubus (ETT) (Davies et al. 1990; Pennant und Walker 1992; Reinhart und Simmons 1994). Verschiedene Studien haben den Einsatz der Larynxmaske während kardiopulmonaler Reanimation untersucht. So konnte Baskett zeigen, dass die Einlage einer LMA durch Pflegepersonal in 97\% innerhalb von 2 Minuten durchgeführt werden konnte, und dass die Beatmung in $85 \%$ aller Fälle suffizient möglich war (Baskett 1994). Die Inzidenz der Aspiration während der Reanimationsmaßnahmen betrug bei Anwendung der LMA 3\%, im Vergleich zur BMV mit einer Häufigkeit von 12\% (Cook und Hommers 2006). Somit wurde die LMA als Alternative zur ETI, oder sogar als primärer Atemweg in die Richtlinien zur CRP der ILCOR (International Liaison Committee on Resuscitation) aufgenommen, wenn keine ausreichenden Kenntnisse und Fertigkeiten zur ETI vorliegen. 
Jedoch gaben in einer Umfrage nur ca. $40 \%$ der englischen Rettungsdienststationen (Ridgway et al. 2004) und nur ca. ein Viertel der innerklinischen Reanimationsteams an (Grayling et al. 2002), derzeit Larynxmasken in der Notfallausstattung mitzuführen. Vergleichbare Zahlen existieren aus einer Umfrage an bayerischen Rettungsdienststandorten, die eine Ausstattung mit LMA von $26 \%$ ermittelte. Daten, wie häufig die LMA gerade unter nicht anästhesiologischem Rettungsdienstpersonal Verwendung findet, liegen jedoch nicht vor. So kommen Cook und Hommers zu dem Schluss, dass die LMA zur Zeit in der Notfallmedizin nicht hinreichend bekannt, nicht ausreichend vorhanden und somit zu wenig eingesetzt wird (Cook und Hommers 2006).

\subsubsection{Die Intubations-Larynxmaske}

Gerade bei Patienten, bei denen eine endotracheale Intubation angestrebt wird, sich aber aus anatomischen, pathologischen oder logistischen Gründen als schwierig erweist, findet die Anwendung der Intubations-Larynxmaske (ILMA oder auch LMAFastrach $^{\text {TM }}$ ) zunehmend Verbreitung. Die von Brain et al. erstmals 1997 vorgestellte ILMA stellt eine Weiterentwicklung der Standard-Larynxmaske als Intubationshilfe dar (Brain et al. 1997a; Brain et al. 1997b). Durch die veränderte Form gelingt die endotracheale Intubation signifikant häufiger, als mit der konventionellen LMA (Langenstein und Möller 1998). Durch die Einbettung eines Metallrohres hat sich die Insertionstechnik gegenüber der LMA geändert. Die ILMA wird am Metallgriff gefasst und in einer Rotationsbewegung streng posterior in Richtung des harten Gaumens eingeführt. Diese Einführtechnik scheint die erfolgreiche Ventilation gerade bei medizinischem Personal, das ungeübt im Umgang mit LMA oder ILMA ist, zu erleichtern (Choyce et al. 2000; Choyce et al. 2001). Der durchschnittliche Ventilationserfolg der ILMA bei schwierigen anatomischen Verhältnissen wird mit initial 90\% und endgültig mit 99\% angegeben. Die „blinde“ Intubation über die ILMA gelingt nach Metaanalyse (Brimacombe 2005) bei normalen und pathologischen Atemwegsverhältnissen im ersten Versuch in $71 \%$ und insgesamt in $90 \%$. Ursprünglich wurde die ILMA zur Anwendung bei Patienten mit schwierigen Atemwegsverhältnissen entwickelt, sie konnte jedoch auch von unerfahrenen Personen erfolgreich zur Ventilation und Intubation bei Patienten eingesetzt werden 
(Choyce et al. 2000; Kurola et al. 2006). Timmermann und Mitarbeiter konnten zeigen, dass die Intubation an einem Intubationstrainer durch unerfahrenes medizinisches Personal schneller und erfolgreicher durchgeführt werden konnte als die LG-TI (Timmermann et al. 2007b).

\subsubsection{Andere extraglottische Atemwege}

Auch andere extraglottische Atemwege (Bowman et al. 1995) wie beispielsweise der Combitubus oder der Larynxtubus haben ihren Stellenwert in der präklinischen Anwendung (Cook und Hommers 2006). Die Anwendungsmöglichkeiten der extraglottischen Atemwegshilfen sowie der Maskenbeatmung sind jedoch, verglichen mit der endotrachealen Intubation, eingeschränkt. Bei Verwendung der extraglottischen Atemwegshilfen besteht kein sicherer Aspirationsschutz und ein erhöhtes Risiko einer Dislokation. Beim Einführen der Atemwegshilfen kann es zu Blutungen und Schwellungen im laryngopharyngealen Bereich kommen, die eine suffiziente Ventilation unmöglich machen können. Die Beatmung ist nur mit geringen Beatmungsdrücken möglich, und es besteht keine Option zur endotrachealen Absaugung von Sekret. Während über einen endotracheal liegenden Tubus eine Medikamentenapplikation möglich ist, besteht diese Möglichkeit bei der Verwendung von extraglottischen Atemwegshilfen nicht.

\subsection{Richtlinien und Standards}

Die Organisation des Rettungsdienstes in der Bundesrepublik Deutschland ist Aufgabe der Bundesländer. So regelt das Niedersächsische Rettungsdienstgesetz (NRettDG vom 02.10.2007) die Belange der Organisation und Durchführung des Rettungsdienstes. Hier heißt es unter $\S 10$ Personal: „Das im Rettungsdienst eingesetzte Personal muss fachlich und gesundheitlich geeignet sein und die erforderliche Zuverlässigkeit besitzen. Es muss entsprechend seiner Verwendung nach einheitlichen Maßstäben aus- und fortgebildet sein und regelmäßig fortgebildet werden." (§10 Abs.1 NRettDG) (NRettDG vom 02.10.2007). Die Regelungen für die 
ärztliche Weiterbildung obliegen den einzelnen Landesärztekammern. Die Ärztekammer Niedersachsen als Körperschaft des öffentlichen Rechts regelt in der „Weiterbildungsordnung der Ärztekammer Niedersachsen vom 27.11.2004“ (Ärztekammer Niedersachsen 2004: Abschnitt A §5 Weiterbildungsordnung der Ärztekammer Niedersachsen vom 27.11.2004) die ärztliche Fort- und Weiterbildung.

Für die Anerkennung der Zusatzbezeichnung Notfallmedizin in Niedersachsen ist der Nachweis einer bestimmten Anzahl an Atemwegssicherungsmaßnahmen nicht erforderlich. Spezielle Anforderungen hinsichtlich der klinischen Fertigkeiten im Bereich des Atemwegmanagements bestehen nicht. Es wird lediglich eine bestimmte Anzahl von notärztlichen Einsätzen unter Supervision gefordert (Ärztekammer Niedersachsen 2004: Abschnitt C Abs. 31 S.160 Weiterbildungsordnung der Ärztekammer Niedersachsen vom 27.11.2004). Die Zusatzbezeichnung Notfallmedizin und die Durchführung der notfallmedizinischen Maßnahmen erfolgt in der Bundesrepublik interdisziplinär. Die Sicherung der Atemwege erfolgt häufig durch Notärzte, die die endotracheale Intubation nicht regelmäßig durchführen (Roessler und Zuzan 2006; Henderson et al. 2004). 


\section{$1.5 \quad$ Ziel der Untersuchung}

Die endotracheale Intubation (ETI) wird als Goldstandard der Atemwegssicherung bezeichnet. Das Erlernen dieses Verfahrens ist jedoch langwierig und setzt eine gewisse Anzahl an Intubationsversuchen voraus. Die alternativen Verfahren der Atemwegssicherung erfüllen zum Teil nicht die Anforderungen nach einem ausreichenden Aspirationsschutz. So stellt sich die Frage, ob es alternative Verfahren in der Atemwegssicherung gibt, die schneller erlernt werden können, als die konventionelle endotracheale Intubation, und gleichzeitig die Vorteile der endotrachealen Intubation, d.h. ausreichenden Aspirationsschutz, bieten.

Vor diesem Hintergrund ist das Ziel dieser prospektiv randomisierten Studie, die Ventilations- und Intubationserfolge bei unterschiedlichen Atemwegssicherungsmaßnahmen und die dafür benötigten Zeitintervalle zu ermitteln. Diese Maßnahmen zur Atemwegssicherung wurden von unerfahrenem medizinischem Personal durchgeführt. Die Anwendung dieser Maßnahmen erfolgte bei Patienten ohne Prädiktoren einer schwierigen Intubation im Rahmen einer elektiv durchgeführten Narkoseeinleitung.

Verglichen werden soll der Ventilationserfolg mit Hilfe der IntubationsLarynxmaske (ILMA-V) gegenüber der Gesichtsmaskenbeatmung (BM-V) einerseits, sowie der Intubationserfolg mit Hilfe der Intubations-Larynxmaske (ILMA-TI) verglichen mit der konventionellen laryngoskopischen Intubation (LG-TI) andererseits. 


\section{$2 \quad$ Material und Methoden}

\subsection{Rekrutierung der Probanden}

Die Durchführung der vorliegenden Studie erfolgte nach Genehmigung der Ethik-Kommission der Medizinischen Fakultät der Universität Göttingen vom 30. Oktober 2005 (Antragsnummer: 26/11/05, vom 24.11.2005).

Aufgenommen in die vorliegende Untersuchung wurden Studentinnen und Studenten des Fachbereichs Medizin, die sich im letzten Ausbildungsjahr (praktisches Jahr) befanden, und das Wahlfach Anästhesiologie gewählt hatten. Während der praktischen Ausbildung im letzten Ausbildungsjahr werden im Rahmen eines Ausbildungscurriculums Atemwegssicherungsmaßnahmen unterrichtet. Diese unterschiedlichen Maßnahmen sollten nun unter kontrollierten Bedingungen durchgeführt werden.

Nicht aufgenommen in die vorliegende Untersuchung wurden Studentinnen und Studenten, die in den letzten 12 Monaten Atemwegssicherungsmaßnahmen außerhalb dieser Studie erlernt oder durchgeführt hatten. Als Maßnahmen zur Atemwegssicherung wurden Gesichtsmaskenbeatmung, laryngoskopisch durchgeführte Intubationen oder die Einlage einer Larynxmaske gewertet.

Die Teilnahme an der Studie erfolgte durch schriftliches Einverständnis der Probanden. Insgesamt wurden 43 Probanden in die unterschiedlichen Techniken der Atemwegssicherung eingewiesen (s. Kap. 2.2.1). Von diesen Probanden konnten dreißig Studentinnen und Studenten endgültig in die Untersuchung aufgenommen werden. Dabei handelte es sich um 17 männliche und 13 weibliche Teilnehmer. Die verbleibenden 13 Studenten konnten nicht in die Untersuchung aufgenommen werden, da die Versuchsdurchgänge nicht komplett durchlaufen wurden, was auf organisatorische Gründe zurückzuführen war.

Bei der vorliegenden Studie handelt es sich um eine prospektive, randomisierte, Crossover-Untersuchung. Die Daten wurden über einen Zeitraum von 10 Monaten, von Januar bis Oktober 2006, erhoben. 


\section{$2.2 \quad$ Studienprotokoll}

\subsubsection{Trainingsphase}

Alle Studierenden nahmen an einer 90 Minuten dauernden Unterrichtseinheit, die die Grundlagen des Atemwegsmanagements beinhaltete, teil.

Der Vortrag behandelte die Darstellung und Veranschaulichung der unterschiedlichen Atemwegssicherungsmaßnahmen d.h. Beutel-Gesichtsmaskenbeatmung (BM-V), laryngoskopisch durchgeführte endotracheale Intubation (LG-TI), Ventilation und Intubation via Intubations-Larynxmaske (ILMA-V, ILMA-TI) (ILMA oder auch LMA- Fastrach ${ }^{\mathrm{TM}}$, LMA, Laryngeal Mask Company, Hemsley on the Thames, Großbritannien).

Im Anschluss wurde jede Technik der Atemwegssicherung durch den Instruktor (Facharzt für Anästhesiologie) am Intubationstrainer (Laerdal Medical AS, Stavanger, Norwegen) vorgeführt.

Weiterhin wurden Techniken zur Optimierung der Intubationsbedingungen und Hilfsmittel der Atemwegssicherung veranschaulicht. Dazu gehörten die Anwendung eines Güdel-Tubus (Güdel-Tubus, Teleflex Medical $\mathrm{GmbH}$, Kernen, Deutschland) und die Zweihand-Maskenbeatmung für die BM-V, sowie das sog. BURP-Manöver, um die endotracheale Intubation zu erleichtern. Das BURP-Manöver (backward, upward, rightward pressure) ist ein Handgriff, mit dem bei einer endotrachealen Intubation die Sicht auf die Glottis verbessert und das Einführen des Tubus erleichtert bzw. erst ermöglicht werden kann. Dabei wird ein nach dorsal, kranial und rechts gerichteter Druck auf den Schildknorpel ausgeübt, so das die direkte Laryngoskopie verbessert oder erst ermöglicht werden kann (Knill 1993).

Die Insertion der ILMA und die Intubation über die liegende ILMA wurden den Probanden demonstriert. Hier wurde nach den von Brimacombe verfassten Handlungsanweisungen verfahren (Brimacombe et al. 1997) (s. Abb. 2.1 bis 2.5).

Bei der Insertion der ILMA befindet sich der Kopf des Patienten in Neutralposition. Der blockbare Cuff der ILMA wird vor der Einlage entlüftet, und mit Gleitmittel auf der Rückseite der ILMA versehen. In der ersten Phase wird die ILMA am Handgriff zwischen Daumen und Zeigefinger gefasst. Die Spitze der Maske wird 
so platziert, dass sie unmittelbar hinter den oberen Schneidezähnen flach am harten Gaumen anliegt. Die Spitze hält Kontakt mit dem harten Gaumen (s. Abb. 2.1).

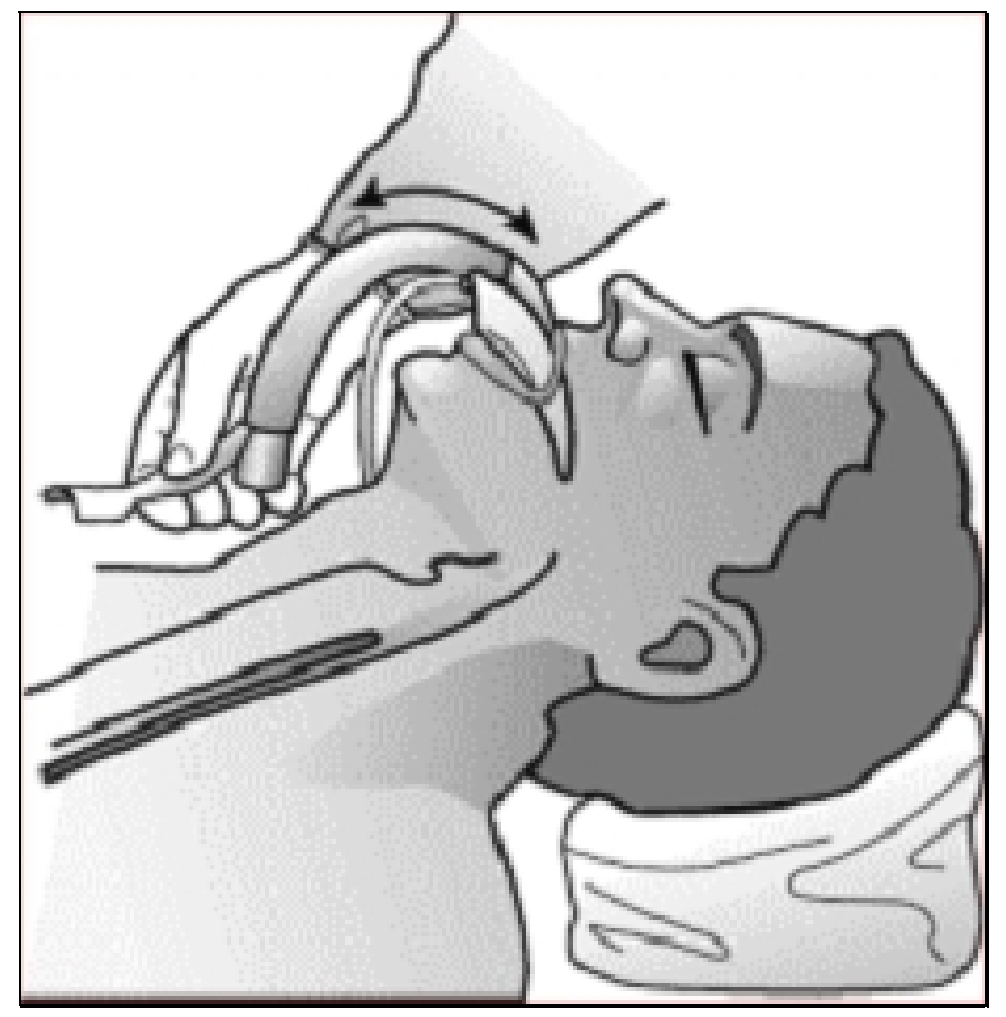

\section{Abb. 2.1: Einführen der ILMA, Phase 1}

Platzierung der ILMA, Phase 1: Die Spitze der Maske wird so platziert, dass sie unmittelbar hinter den oberen Schneidezähnen am harten Gaumen anliegt (aus LMA-Fastrach Anleitungshandbuch 2002, S.8 - mit freundlicher Genehmigung der LMA Deutschland $\mathrm{GmbH}$, Bonn).

Vor dem Platzieren der ILMA sollte der gebogene Anteil der Larynxmaske auf dem Kinn des Patienten aufliegen, somit wird der Kontakt zum harten Gaumen gehalten (s. Abb. 2.2). 


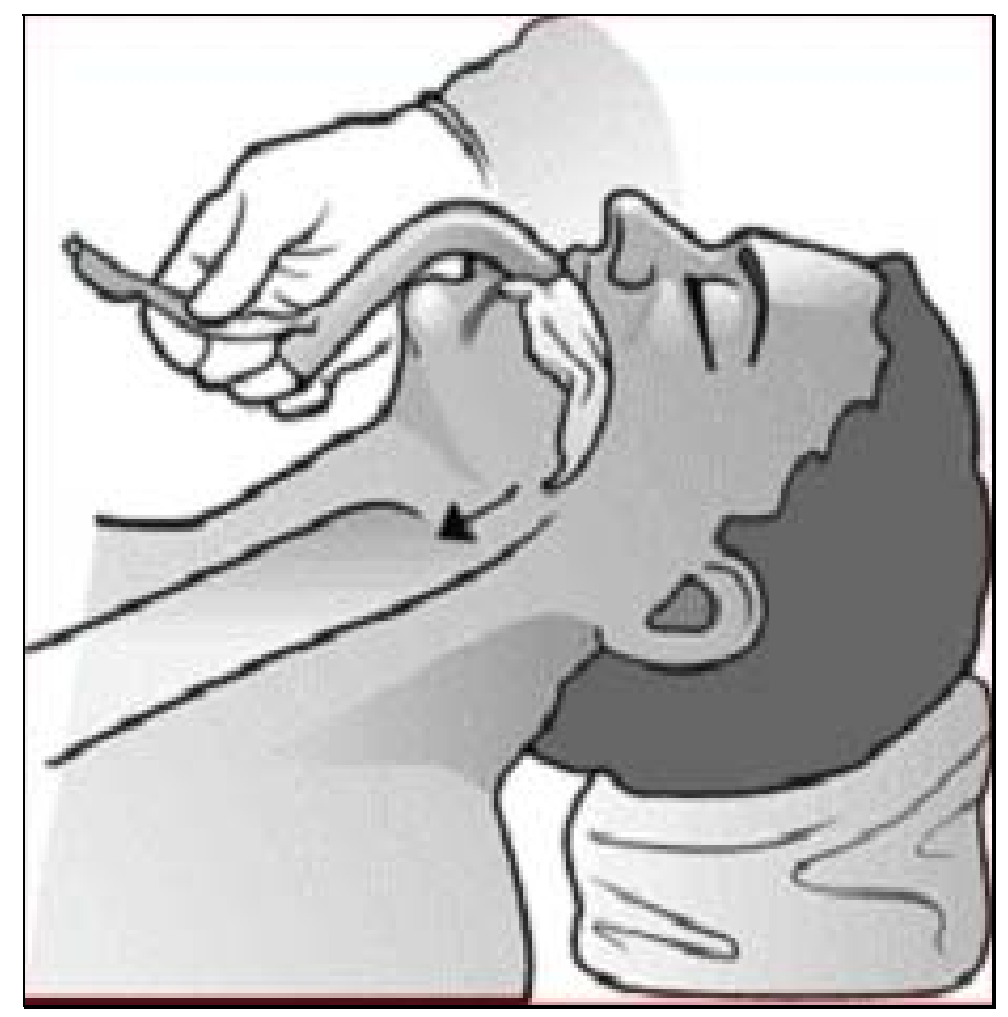

\section{Abb. 2.2: Einführen der ILMA, Phase 2}

Platzierung der ILMA, Phase 2: Der Kontakt zum harten Gaumen wird gehalten (aus LMAFastrach Anleitungshandbuch 2002, S.8 - mit freundlicher Genehmigung der LMA Deutschland $\mathrm{GmbH}$, Bonn).

Die ILMA wird nun am Handgriff gefasst, und in einer gleichförmigen zirkulären Bewegung in den Hypopharynx vorgeschoben, dabei wird ständig Kontakt mit dem harten Gaumen gehalten. Der Handgriff der ILMA sollte nicht als Hebelarm benutzt werden, da es sonst zu Verletzungen oropharyngealer Strukturen kommen kann. Nach erfolgter Einlage kann der Cuff der ILMA geblockt werden. Hierbei sollte, nach den Angaben des Herstellers, ein Cuffdruck von $60 \mathrm{cmH}_{2} \mathrm{O}$ nicht überschritten werden (s. Abb. 2.3). 


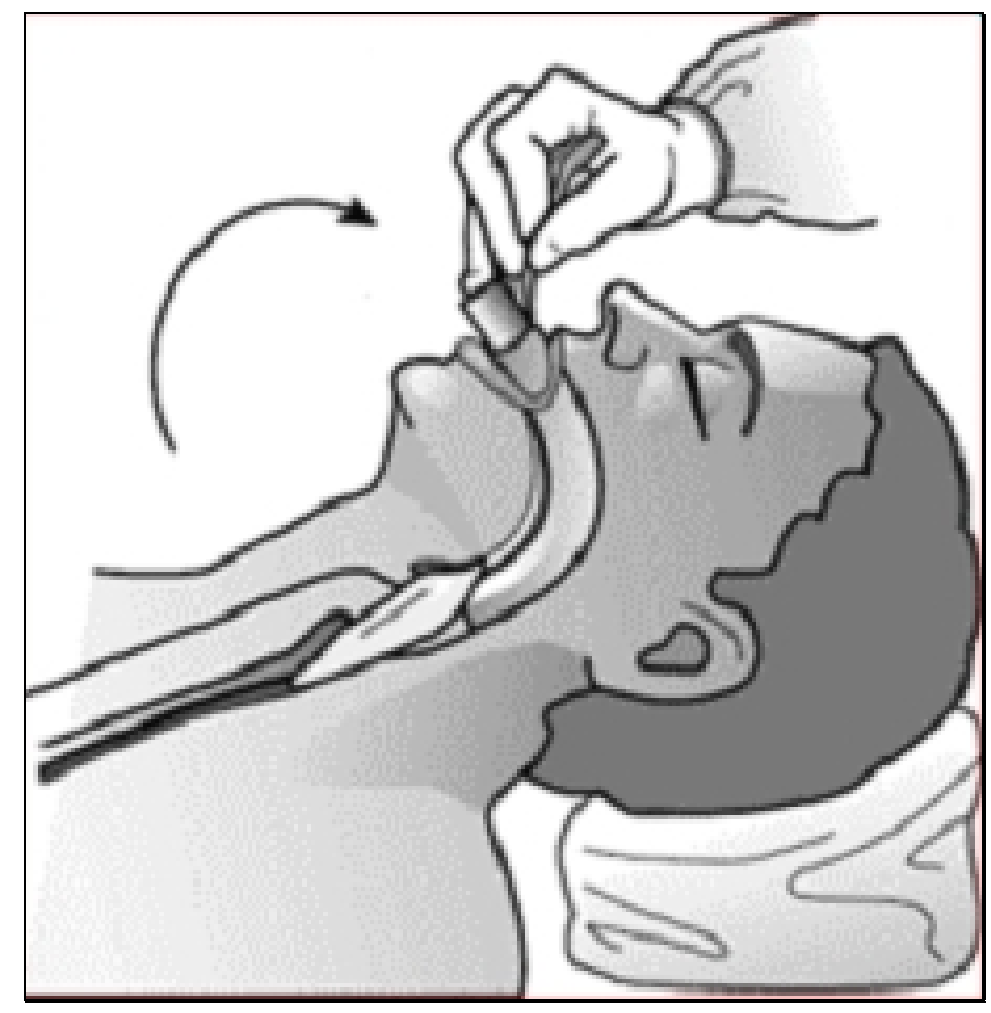

\section{Abb. 2.3: Einführen der ILMA, Phase 3}

Platzierung der ILMA, Phase 3: Vorschieben der ILMA in den Hypopharynx (aus LMAFastrach Anleitungshandbuch 2002, S.9 - mit freundlicher Genehmigung der LMA Deutschland $\mathrm{GmbH}$, Bonn).

Ist die Insertion der Intubations-Larynxmaske abgeschlossen, und der Cuff der ILMA geblockt, so kann die Beatmung des Patienten erfolgen, indem die Maske mit einem Beatmungsgerät oder einem Beatmungsbeutel konnektiert wird. Bei Bedarf kann nun die endotracheale Intubation via ILMA erfolgen. Der mit Gleitmittel versehene ETT wird in den Tubus der ILMA eingeführt, dabei wird der Griff der ILMA fest gehalten. Um das Gleitmittel gleichmäßig zu verteilen, wird der Tubus in der ILMA mit einer rotierenden Bewegung hin und her bewegt. Auf dem ETT befinden sich Markierungen zur Ausrichtung der Spitze. Beim Einsatz des ETT muss die schwarze, entlang des Tubus verlaufende Linie zum Handgriff der Maske zeigen. Zu diesem Zeitpunkt sollte die querverlaufende Markierung, welche das Auftreten der Tubusspitze auf den Epiglottisheber indiziert, nicht im Tubus der ILMA verschwinden (s. Abb. 2.4). 


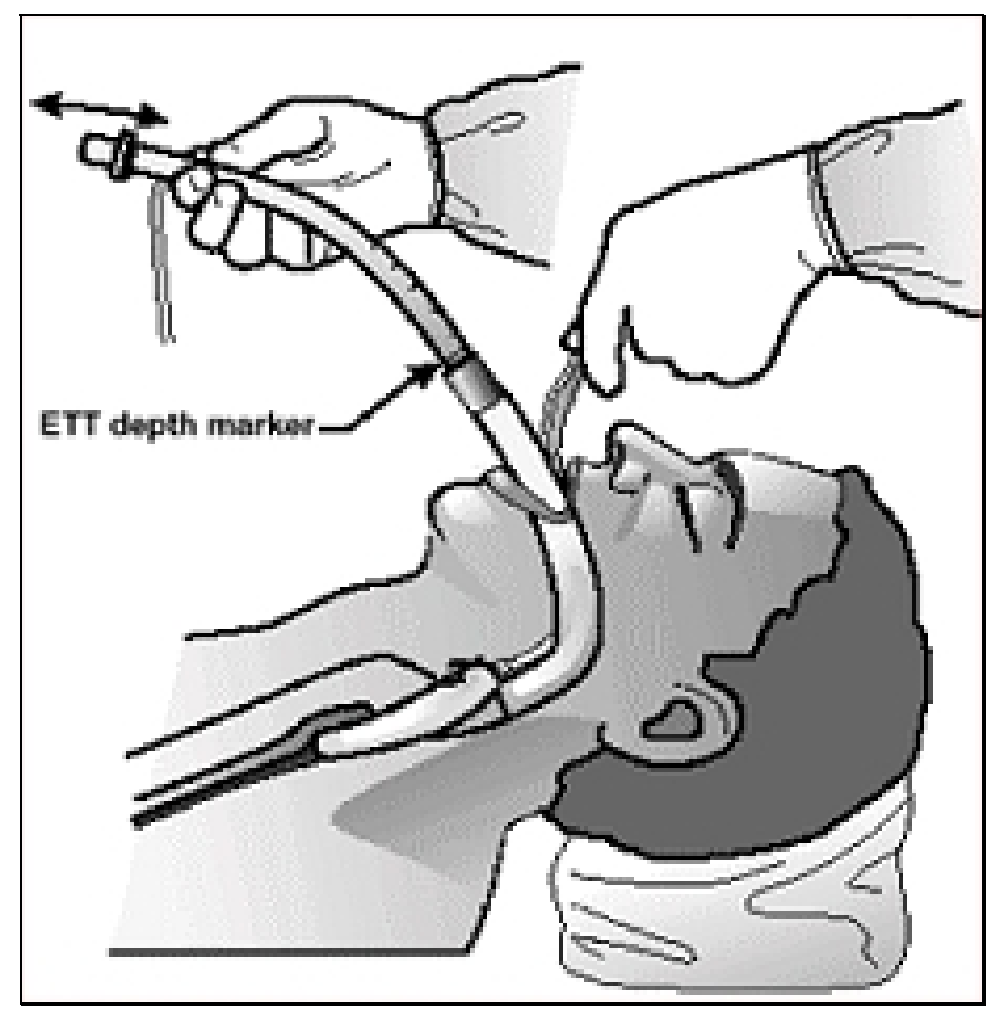

Abb. 2.4: Intubation über die liegende ILMA, Phase 1

Intubation über die liegende ILMA, Phase 1: Der Handgriff der ILMA wird fixiert, und der ETT wird entsprechend den Markierungen vorgeschoben (aus LMA-Fastrach Anleitungshandbuch 2002, S. 11 - mit freundlicher Genehmigung der LMA Deutschland GmbH, Bonn).

Ist nun die querverlaufende Markierung des ETT auf dem Niveau der ILMA angelangt, wird der Hangriff der ILMA einige Millimeter angehoben, dies bewirkt eine Kranialverlagerung des Hypopharynx (Chandi-Manöver), so dass die endotracheale Intubation erleichtert wird. Liegt die ILMA richtig, sollte die Intubation möglich sein, ohne größeren Widerstand überwinden zu müssen. Der Tubus wird vorgeschoben, während die ILMA mit der anderen Hand fixiert wird. Der Cuff des ETT kann nun geblockt werden (s. Abb. 2.5). 


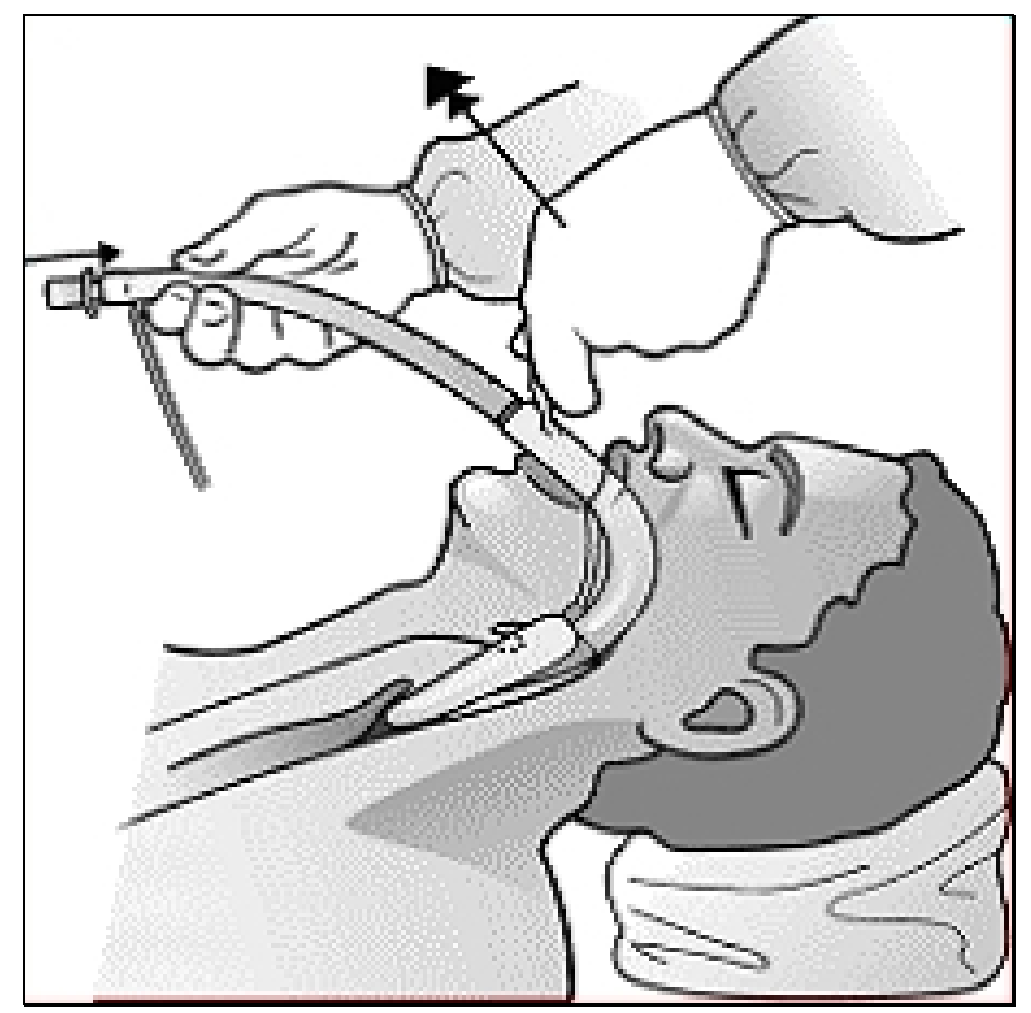

Abb. 2.5: Intubation über die liegende ILMA, Phase 2

Intubation über die liegende ILMA, Phase 1: Der Handgriff der ILMA wird angehoben, damit wird ein Vorschieben des ETT erleichtert (aus LMA-Fastrach Anleitungshandbuch 2002, S.11 - mit freundlicher Genehmigung der LMA Deutschland $\mathrm{GmbH}$, Bonn).

Weiterhin wurden Möglichkeiten zur Verbesserung der Intubationsbedingungen demonstriert, dazu gehören das sog. „Up-and-down“-Manöver und das „Chandi“Manöver (s.Abb. 2.5).

Kommt es nach Einlage der ILMA zu einer nach kaudal umgeschlagenen Epiglottis, so kann über die Intubations-Larynxmaske keine endotracheale Intubation mehr erfolgen. Dies macht sich durch einen federnden Widerstand beim Einführen des Tubus bemerkbar. Mit Hilfe des sog. „Up-and-down“-Manövers kann diese Fehllage korrigiert werden. Der Endotrachealtubus wird zunächst entfernt, dann wird die ILMA in geblocktem Zustand ca. $6 \mathrm{~cm}$ zurückgezogen, und erneut vorgeschoben. Dieses „Up-and-down“-Manöver hat sich beim Aufrichten einer nach kaudal umgeschlagenen Epiglottis als wirkungsvoll erwiesen.

Das Chandi-Manöver hingegen erleichtert die endotracheale Intubation über die ILMA. Der Handgriff der ILMA wird nach dem Einführen nach dorsal gedrückt, so dass sich das kaudale Ende der ILMA nach ventral bewegt und der Pharynx um 
einige Millimeter angehoben wird. Das Chandi-Manöver erhöht die Dichtigkeit und bringt den Tubus in optimale Position zur trachealen Achse. Somit wird die endotracheale Intubation über die liegende Intubations-Larynxmaske erleichtert.

Die potentiellen Fehllagen der ILMA wurden ebenfalls am Modell demonstriert. Als Fehllage wird die nach kaudal umgeschlagene Epiglottis angesehen, die mit Hilfe des „Up-and-down“-Manövers, wie oben beschrieben, behoben werden kann. Ein weiterer Fehler ist das Einführen einer zu kleinen ILMA. Ist dies der Fall, so spürt man $3 \mathrm{~cm}$ hinter der 15-cm-Quermarkierung einen gewissen Widerstand. In diesem Fall ist die Epiglottis außerhalb der Reichweite des Epiglottishebers. Dies indiziert die Verwendung der nächst größeren Maske. Die gleiche Situation kann auftreten, wenn der Larynx während der Insertion der ILMA nach kaudal gedrückt wird. Dies kann ein Resultat unzureichender Narkosetiefe oder falscher Einführtechnik sein. Ist die ILMA hingegen zu groß, so spürt man beim Einführen des Tubus bereits einen Widerstand, wenn die Quermarkierung des Endotrachealtubus noch sichtbar ist. In diesem Fall befindet sich der Epiglottisheber hinter den Arytenoiden. Wenn hier versucht wird, gegen diesen Widerstand $\mathrm{zu}$ intubieren, so gelangt der ETT mit großer Wahrscheinlichkeit in den Ösophagus.

Nach erfolgter theoretischer Einweisung wurden die Probanden aufgefordert, die unterschiedlichen Ventilations- und Intubationstechniken am Intubationstrainer zu üben und zu erlernen. Es mussten je Technik mindestens drei erfolgreiche Versuche absolviert werden, bevor die Probanden in die Studienphase aufgenommen werden konnten. Die verwendeten Atemwegstechniken waren die Gesichtsmaskenbeatmung (BM-V), die endotracheale Intubation (LG-TI), die Ventilation und Intubation über die Intubations-Larynxmaske (ILMA-V, ILMA-TI).

Die Trainingsphase wurde mit der Demonstration der unterschiedlichen Atemwegstechniken am anästhesierten Patienten durch den Instruktor abgeschlossen.

\subsubsection{Rekrutierung der Patienten}

Patienten, die in die Untersuchung aufgenommen wurden, mussten volljährig und geschäftsfähig sein. Es wurden Patienten mit elektiven operativen Eingriffen, ohne signifikante Vorerkrankungen (ASA Status I und II) oder Prädiktoren 
schwieriger Atemwegsverhältnisse, in die Untersuchung eingeschlossen. Dabei erfolgte die Rekrutierung der Patienten aus den unterschiedlichen operativen Disziplinen (s. Tab. 2.1).

\begin{tabular}{|lccc|}
\hline operative Disziplin & $\begin{array}{c}\text { Patienten } \\
\text { männlich, } \mathbf{n}\end{array}$ & $\begin{array}{c}\text { Patienten } \\
\text { weiblich, }\end{array}$ & $\begin{array}{c}\text { Gesamtzahl, } \\
\text { Summe }\end{array}$ \\
\hline Urologie/ Gynäkologie & 34 & 10 & 44 \\
\hline $\begin{array}{l}\text { Allgemeinchirurgie/ Thorax- } \\
\text { Herz-Gefäßchirurgie }\end{array}$ & 14 & 21 & 35 \\
\hline Unfallchirurgie/ Orthopädie & 19 & 22 & 41 \\
\hline $\begin{array}{l}\text { HNO/ Mund- Kiefer- } \\
\text { Gesichtschirurgie }\end{array}$ & 9 & 13 & 30 \\
\hline Neurochirurgie/ Augenheilkunde & 16 & 14 & 8 \\
\hline Dermatologie & 7 & 1 & 180 \\
\hline Summe & 99 & 81 & 22 \\
\hline
\end{tabular}

Tab. 2.1: Patientenzahlen bezogen auf die operativen Disziplinen

Die Beurteilung der Atemwegsverhältnisse jedes einzelnen Patienten erfolgte im Rahmen der Prämedikationsvisite durch den jeweiligen Anästhesisten. Als Beurteilungskriterien wurden die Klassifikation nach Mallampati (Samsoon und Young 1987), die Überstreckbarkeit im Atlantookzipitalgelenk, die Protrusionsfähigkeit des Unterkiefers und die Größe der Mundöffnung herangezogen.

Bei der Klassifikation nach Mallampati wird der aufrecht sitzende Patient aufgefordert, bei neutraler Position des Kopfes den Mund so weit wie möglich zu öffnen und die Zunge so weit wie möglich herauszustrecken. Dabei wird die Sichtbarkeit der oropharyngealen Strukturen beurteilt. Zwischen der Einsehbarkeit des Rachens nach dem Mallampati-Score und der Leichtigkeit der endotrachealen Intubation besteht eine positive Korrelation (Samsoon und Young 1987). Die Einteilung erfolgt in vier Grade, wobei Grad eins eine vermeintlich einfache 
endotracheale Intubation und Grad vier eine vermeintlich schwierige endotracheale Intubation impliziert. Grad eins erhalten Patienten, deren weicher Gaumen, Uvula und seitliche Tonsillenbögen voll einsehbar sind. Bei Grad zwei sind die seitlichen Tonsillenbögen nicht mehr sichtbar. In Grad drei werden Patienten eingeteilt, deren weicher Gaumen und Uvula nur noch teilweise sichtbar sind. Bei Grad vier ist ausschließlich der harte Gaumen einsehbar. Kein Patient, der in die vorliegende Untersuchung aufgenommen wurde, hatte eine Mallampati- Klassifikation größer als zwei.

Eine weitere Möglichkeit, die Atemwegsverhältnisse zu beurteilen, ist die Fähigkeit der Reklination des Kopfes, also die Überstreckbarkeit im Atlantookzipitalgelenk. Ist die Überstreckung ausreichend, können Mund, Pharynx und Larynx in eine gemeinsame Achse gebracht werden, was die Sichtverhältnisse während der endotrachealen Intubation verbessert (Bellhouse und Dore 1988). Eine Reduktion der Überstreckbarkeit um ca. ein Drittel (normalerweise ist eine Überstreckbarkeit um ca. $35^{\circ}$ möglich) führt dazu, dass das Risiko einer schwierigen Intubation um mehr als 20\% steigt (Bellhouse und Dore 1988).

Der thyreomentale Abstand, Test nach Patil (Patil et al. 1983) ergibt sich aus der Distanz zwischen Innenkante der Mandibula und Incisura thyroidea superior, der durchschnittlich 6,5 bis 7,0 cm beträgt. Die Messung zwischen der Innenkante der Mandibula und Oberkante des Sternums, bei geschlossenem Mund und maximaler Extension im Atlantookzipitalgelenk, wird als Test nach Savva (Savva 1994) bezeichnet und beträgt im Mittel 12,5 bis $13,5 \mathrm{~cm}$. Kein Patient hatte einen kleineren thyreomentalen Abstand (Test nach Patil) als 6,0 cm.

Die Fähigkeit zur Protrusion, d.h. das „Vorschieben des Unterkiefers“, kann in drei Grade eingeteilt werden. Grad eins beinhaltet, dass die untere Zahnreihe horizontal vor die obere geschoben werden kann. Grad zwei, dass die untere Zahnreihe nur in Höhe der oberen bewegt werden kann, und Grad drei, dass es nicht möglich ist, die untere Zahnreihe in Okklusionsstellung zu bringen. Bei keinem Patienten war die Protrusionsfähigkeit des Unterkiefers kleiner als $5 \mathrm{~mm}$.

Die Mundöffnung der Patienten sollte ausreichend groß sein. Nach Vaughan (Latto und Vaughan 1997) sollte der Patient in der Lage sein, bei maximal geöffnetem Mund die mittleren drei Finger seiner rechten Hand zwischen die 
Zahnreihen bzw. die Alveolarkämme zu bringen. Kein Patient hatte eine kleinere Mundöffnung als $3,0 \mathrm{~cm}$.

War der Body Mass Index größer als $35 \mathrm{~kg} / \mathrm{m}^{2}$ oder wurden Intubationsprobleme bei vorangegangenen Narkosen vom Patienten geschildert, so wurde der Patient ebenfalls nicht in die Studie aufgenommen.

Jeder Proband sollte die Ventilation und Intubation bei sechs Patienten, im Rahmen einer routinemäßigen Narkoseinduktion durchführen, drei mit jeder Technik. Die Randomisierung erfolgte paarweise, so dass nach jeder erstausgeführten Technik die jeweilige Alternative durchgeführt wurde.

Die Studie wurde in zwei Phasen unterteilt. In der ersten Phase führten die Teilnehmer die zugewiesene Technik durch. Falls diese scheiterte, konnte der Instruktor (Fach- oder Oberarzt für Anästhesiologie) entscheiden, ob die alternative Technik für einen weiteren Versuch angewendet werden durfte. Diese Entscheidung basierte auf Kriterien wie der hämodynamischen Stabilität, der guten Oxygenierung und dem Fehlen selbst leichter traumatischer Veränderungen in den oberen Atemwegen, wie beispielsweise blutiges Sekret am Laryngoskop oder der ILMA.

Die Vitalparameter wurden kontinuierlich während des gesamten Versuchsdurchganges registriert. Als Abbruchkriterien wurden eine periphere Sauerstoffsättigung kleiner als 95\%, oder Abweichungen der Herzfrequenz bzw. des systolischen Blutdrucks von mehr als $20 \%$ des Ausgangswertes definiert. Alle Versuchsdurchgänge erfolgten unter Supervision eines erfahrenen Anästhesisten, der mit dem Studienprotokoll vertraut war.

\subsubsection{Studienphase I}

Die Patienten wurden drei Minuten präoxygeniert $\left(\mathrm{FiO}_{2}, 1,0\right)$, anschließend erfolgte die Narkoseinduktion mit $2 \mu \mathrm{g} / \mathrm{kg}$ Fentanyl und $2 \mathrm{mg} / \mathrm{kg}$ Propofol. Die Narkose wurde mit einer 1,5 bis 2,5- prozentigen endexspiratorischen Sevoflurankonzentration weitergeführt. Bei ausreichender Narkosetiefe übernahm der Instruktor die Maskenbeatmung. War die Maskenbeatmung problemlos möglich, wurde an den Probanden übergeben, der dann die zugewiesene Technik 
durchführte. War die Maskenbeatmung durch den erfahrenen Instruktor nicht oder nur unzureichend möglich, wurde der Patient aus der Untersuchung ausgeschlossen.

Die BM-V erfolgte mit einer Beatmungsmaske (Fa. Intersurgical, Sankt Augustin, Deutschland), die an das Kreissystem des Narkosegerätes angeschlossen war. Der Frischgasfluss betrug 10l/min $\left(\mathrm{FiO}_{2} 1,0\right)$. Für jeden Patienten wurde eine Gesichtsmaske in entsprechender Größe verwendet. Patienten wurden mit der Beatmungsmaske der Größe 5 beatmet, und Patientinnen mit der Beatmungsmaske der Größe 4. Initial wurde die BM-V ohne Hilfsmittel oder unterstützende Maßnahmen durchgeführt, die aber von den Probanden angefordert werden konnten. Zum einen war es möglich, einen Güdel-Tubus (Fa. Teleflex Medical GmbH, Kernen Deutschland), in entsprechender Größe, zur Erleichterung der Ventilation anzufordern. Güdel-Tuben wurden in der Größe 4 für Patienten und der Größe 3 für Patientinnen angewendet. Zum anderen konnte die Zwei- Hand- Maskenbeatmung durch den Probanden angefordert werden. In diesem Fall übernahm der Instruktor die Bedienung des Beatmungsbeutels, und der Proband hielt die Gesichtsmaske mit beiden Händen.

Die ILMA wurde vor Insertion mit Endogel ${ }^{\circledR}\left(\right.$ Endogel $^{\circledR}$, Farco-Pharma GmbH, Köln, Deutschland) an der Spitze der Larynxmaske gleitfähig gemacht. Die Größe 4 wurde für alle weiblichen, die Größe 5 für alle männlichen Patienten verwendet. Nach Insertion der ILMA wurde in den Cuff der Larynxmaske gerade soviel Luft insuffliert, dass die Larynxmaske luftdicht abgeschlossen war, maximal war ein Druck von 60 $\mathrm{cm} \mathrm{H}_{2} \mathrm{O}$ zulässig. Die vom Hersteller empfohlenen Insufflationsmengen von $30 \mathrm{ml}$ Luft für die ILMA der Größe 4, und 40 ml Luft für die ILMA der Größe 5, wurden nicht überschritten. Die Intubations-Larynxmaske wurde nach Insertion an das Kreissystem des Narkosegerätes angeschlossen. Falls keine suffiziente Ventilation hergestellt werden konnte, durfte die wiederholte Einlage versucht werden, sofern dies innerhalb des vorgegebenen Zeitlimits möglich war.

Eine ausreichende Ventilation für die BM-V und ILMA-V wurde definiert, als Rechtecksignal in der Kapnographie mit einem endtidalen $\mathrm{CO}_{2}$-Partialdruck von 3 $\mathrm{kPa}$.

Die Zeit für einen Versuchsdurchgang (Ventilation oder Intubation) betrug 60 Sekunden. Waren die Gesichtsmaskenbeatmung oder die Insertion der ILMA in der vorgegebenen Zeit nicht möglich, übernahm der Instruktor die Ventilation des 
Patienten für mindestens 90 Sekunden, bevor mit dem Versuchsdurchgang fortgefahren werden konnte. War die BM-V in dem vorgegebenen Zeitintervall nicht möglich, so wurde der Versuch nicht gewertet, und der Proband führte dann die LGTI durch. War die ILMA-V nicht zufriedenstellend möglich, wurde auch dieser Versuch nicht gewertet, und der Proband führte unverzüglich die ILMA-TI durch. Wenn die Einlage der ILMA selbst nicht möglich war, wechselte der Proband die Technik (BM-V und LG-TI), und die ILMA-V sowie die ILMA-TI wurden als erfolglos gewertet.

War die Ventilation (BM-V oder ILMA-V) etabliert, so erfolgte die neuromuskuläre Blockade des Patienten, mit $0,6 \mathrm{mg} / \mathrm{kg}$ Rocuronium intravenös. Während der Anschlagzeit von ca. 90 Sekunden wurde die Ventilation fortgeführt, im Anschluss erfolgte nach Studienprotokoll die Intubation.

Für die direkte Laryngoskopie wurde ein Laryngoskop mit einem MaclntoshSpatel der Größe 3 angewendet. Alternativ konnte ein Maclntosh-Spatel der Größe 4 angefordert werden. Die Intubation erfolgte mit einem endotrachealen Tubus (Mallinckrodt Medical, Athlone, Irland), der einen Innendurchmesser (ID) von 7,5 mm bzw. 8,0 mm hatte, und in den ein Führungsstab bis zur Spitze des Tubus eingeführt wurde. Nach Aufforderung des Probanden konnte ggf. das BURP-Manöver durchgeführt werden.

Die ILMA-TI erfolgte mit einem blockbaren Spiraltubus (ID 7,5 mm bzw. 8,0 $\mathrm{mm}$ ), der eine weiche Silikonspitze und Längenmarkierung (LMA-Fastrach ${ }^{\mathrm{TM}}$ Endotracheal Tube, Laryngeal Mask Company, Hemsley on the Thames, Großbritanien) hatte. Der Endotrachealtubus konnte an das Kreissystem des Narkosegerätes zur Ventilation angeschlossen werden.

Konnte die Intubation nicht innerhalb von 60 Sekunden vorgenommen werden, erfolgte eine erneute Ventilation für 90 Sekunden über die Gesichtsmaske bzw. die ILMA. Im Anschluss wurde ein zweiter Intubationsversuch durchgeführt. War die Intubation erfolgreich, wurde die Zeit des ersten und zweiten Intubationsversuchs addiert, so ergab sich eine Gesamtzeit aus beiden Versuchen. War der zweite Intubationsversuch innerhalb der vorgegebenen Zeit ebenfalls nicht möglich, wurde der Durchgang als misslungen gewertet. 


\subsubsection{Studienphase II}

War es nach zwei Versuchsdurchgängen für den Probanden nicht möglich, die Intubation in der vorgegebenen Zeit durchzuführen, wurde die Intubationstechnik gewechselt, und ein weiterer Versuch konnte erfolgen. So wurde bei misslungener laryngoskopischer Intubation die ILMA-TI durchgeführt, und bei misslungener ILMATI die LG-TI. 


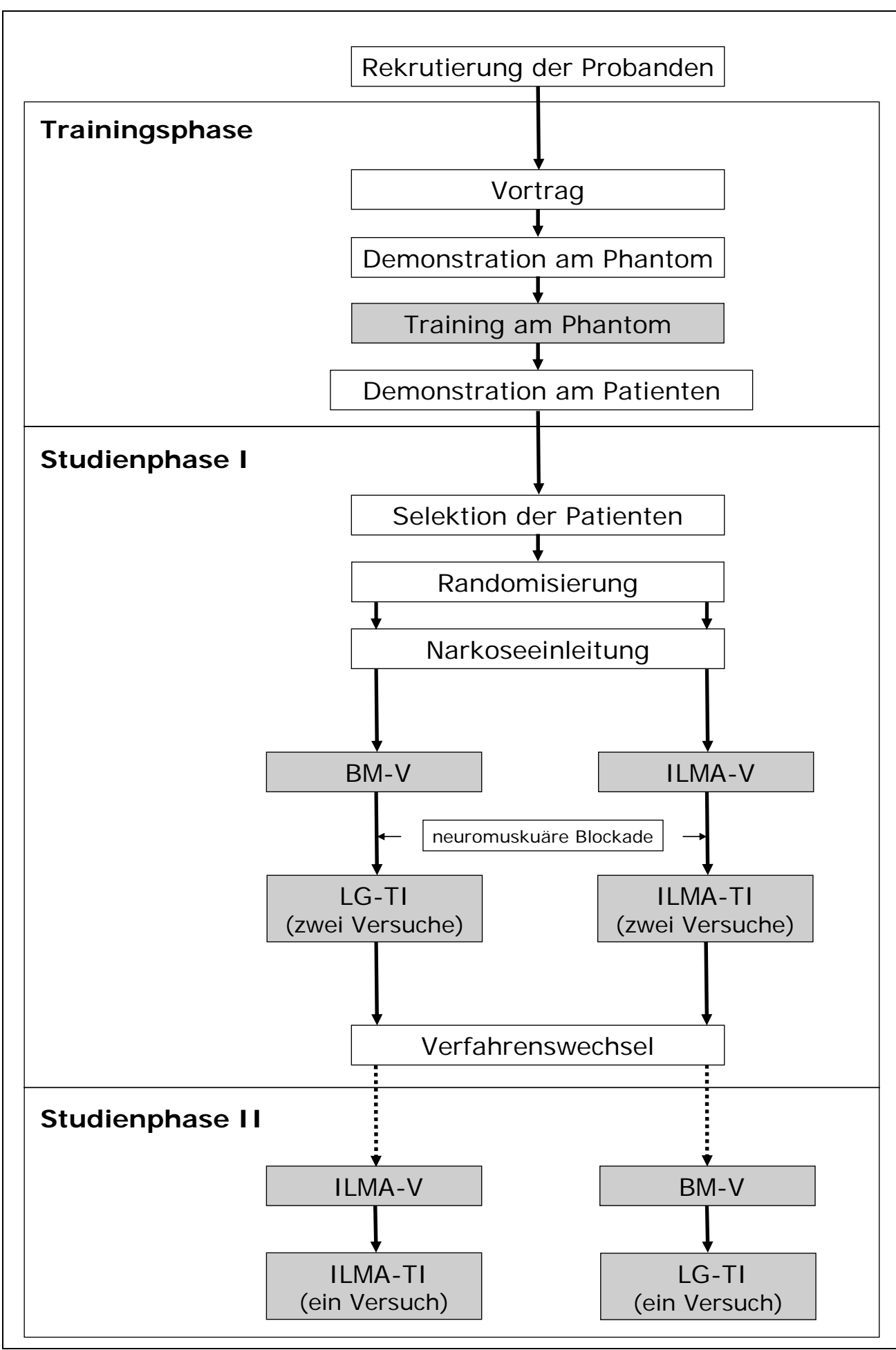

\section{Abb. 2.6: Graphische Darstellung des Versuchsablaufs}

Abkürzungen: BM-V, Ventilation via Gesichsmaske; LG-TI, laryngoskopisch durchgeführte endotracheale Intubation; ILMA-V, Ventilation via Intubationslarynxmaske; ILMA-TI, Intubation via Intubationslarynxmaske. 


\subsection{Datenerfassung und Auswertung}

Ziel der vorliegenden Untersuchung war es die Erfolgsraten und Zeiten der unterschiedlichen Atemwegssicherungsmaßnahmen für die erfolgreiche Durchführung der Ventilation und Intubation zu vergleichen.

Die Zeit für eine erfolgreiche Ventilation (BM-V bzw. ILMA-V) wurde definiert als Zeitspanne zwischen Übergabe der Beatmung vom Instruktor an den Probanden bis zum ersten validen Kapnographie-Signal. War die Ventilation innerhalb der vorgegebenen Zeit nicht möglich, wurde der Versuchsdurchgang mit 60 Sekunden gewertet und beendet.

Die Gesamtzeit der Intubationsversuche (LG-TI bzw. ILMA-TI) wurde aus der Addition der Einzelversuche bis zum Erfolg ermittelt (also max. 120 Sekunden bei zwei Versuchsdurchgängen). Gewertet wurde auch hier die Zeitspanne vom Beginn der Intubation bis zum ersten validen Kapnographie-Signal.

Für jeden Versuchsdurchgang wurden die verwendeten Hilfsmittel und unterstützenden Maßnahmen, wie in Kap. 2.2.1 beschrieben, dokumentiert.

Die Ventilations- und Intubationsversuche wurden bei 180 Patienten aus unterschiedlichen operativen Fachabteilungen durchgeführt. Für die Patienten wurden unterschiedliche demographische Daten erhoben, dazu zählten das Geschlecht, Körpergröße und -Gewicht sowie das Alter der Patienten. Für jeden Patienten wurde der Body Mass Index errechnet.

\subsection{Statistische Analysen}

Die Daten für die Ventilationen und Intubationen wurden mit einem Datenverarbeitungsprogramm aufgezeichnet (Excel 2002, Microsoft Corp, Redmond, Wash., USA) und mit dem Statistikprogramm SPSS (SPSS 12.0.1, SPSS Inc, Chicago, III., USA) ausgewertet. 
Der Intubations- bzw. der Ventilationserfolg wurde mit dem Wilcoxon-Test verglichen. Für jeden Durchlauf kam der McNemar-Test zur Anwendung. Die Gesamtzeit für die Ventilation und Intubation wurde mit der Varianzanalyse für wiederholte Messungen verglichen ( $r m$ ANOVA = repeates-measure of variance). Die Daten wurden auf Sphärizität geprüft. Der Greenhouse-Gerisser-Test wurde zur Adjustierung der Freiheitsgrade benutzt. Die Zeiten für eine erfolgreiche Ventilation bzw. Intubation wurden mit Hilfe der Kaplan-Meier-Kurven dargestellt.

Demographische und deskriptive Daten der Patienten (Alter, Gewicht, Größe und $\mathrm{BMI}$ ) der Patienten wurden für jede Gruppe verglichen mit univariater Varianzanalyse (ANOVA) auf Signifikanz geprüft. Das Geschlechterverhältnis wurde mit dem $X^{2}-$ Test getestet. Das statistische Signifikanzniveau betrug $\alpha<0.05$. 


\section{$3 \quad$ Ergebnisse}

\subsection{Demographische Daten}

Insgesamt wurden 186 Patienten in die Studie aufgenommen. Sechs Patienten konnten nach Einleitung der Narkose nicht eingeschlossen werden. Davon konnten drei Patienten vom Testleiter nur unter Schwierigkeiten ventiliert werden, und bei weiteren drei Patienten war der systolische Blutdruck außerhalb der Toleranzgrenzen.

Es wurden keine signifikanten Unterschiede zwischen den beiden PatientenIntubations-Gruppen für die Items Alter, Körpergewicht, Größe, BMI oder Geschlecht gefunden (Tab. 3.1).

Fünfzehn Teilnehmer des Probandenkollektivs begannen mit der Gesichtsmaskenbeatmung gefolgt von der laryngoskopischen Intubation, und fünfzehn Teilnehmer begannen mit der ILMA-Ventilation gefolgt von der ILMAIntubation.

\begin{tabular}{|lcc|c|c|}
\hline & $\begin{array}{c}\text { BM-V I } \\
\text { LG-TI }\end{array}$ & $\begin{array}{c}\text { ILMA-V I } \\
\text { ILMA-TI }\end{array}$ & p-Wert & $\begin{array}{c}\text { ANOVA } \\
\left(\mathbf{F}_{1,178}\right)\end{array}$ \\
\hline Anzahl der Patienten & 90 & 90 & n.a. & n.a. \\
\hline Geschlecht männlich, n (\%) & $37(41 \%)$ & $44(49)$ & $0,29^{\mathrm{a}}$ & n.a. \\
\hline Alter, Mittelwert (SD), [Jahre] & $53,9 \pm 19,2$ & $50,2 \pm 15,3$ & $0,16^{\mathrm{b}}$ & 2,0 \\
\hline Größe, Mittelwert (SD), [cm] & $169,8 \pm 10,4$ & $172,0 \pm 9,2$ & $0.14^{\mathrm{b}}$ & 2,2 \\
\hline Gewicht, Mittelwert (SD), [kg] & $76,3 \pm 15,9$ & $76,5 \pm 13,4$ & $>0,9^{\mathrm{b}}$ & $<1,0$ \\
\hline BMI, Mittelwert (SD), [kg m $\left.{ }^{-2}\right]$ & $26,4 \pm 4,9$ & $25,8 \pm 3,9$ & $0,9^{\mathrm{b}}$ & 0,35 \\
\hline
\end{tabular}

\section{Tab. 3.1: Demographische und deskriptive Daten}

Abkürzungen: BMI, Body Mass Index; BM-V, Ventilation via Gesichtsmaske; ILMA-V, Ventilation via Intubations-Larynxmaske; LG-TI, laryngoskopisch durchgeführte endotracheale Intubation; ILMA-TI, Intubation via Intubations-Larynxmaske, statistische Tests $a, \chi_{(1)}^{2}=1,1 ; b$, ANOVA $\left(F_{1,178}\right)$. 


\subsection{Atemwegssicherungsmaßnahmen}

\subsubsection{Ventilationsmaßnahmen}

Die ILMA-V konnte bei 88 von 90 (97,8\%) Patienten signifikant häufiger erfolgreich durchgeführt werden, als die Gesichtsmaskenbeatmung, die bei 77 von $90(85,6 \%, p<0,05)$ Patienten erfolgreich war. Die benötigte Zeit bis zur erfolgreichen Ventilation war mit der ILMA-V signifikant kürzer, als mit der Gesichtsmaskenbeatmung (35,6 $\pm 13,5$ s versus $44,3 \pm 16,1 s, F=22,8, p<0,01)$. Es konnte weder ein signifikanter Einfluss bezüglich des Messzeitpunktes $(F=0,3$, $p=0,73$, noch eine signifikante Interaktion bezüglich der verwendeten Methode $(F=1,0, p=0,38)$ festgestellt werden (s. Tab. 3.2).

\begin{tabular}{|lcc|}
\hline & BM-V & ILMA-V \\
\cline { 2 - 3 } $\begin{array}{l}\text { Durchgang, } \\
\text { Anzahl der Patienten }\end{array}$ & $\begin{array}{c}\text { Erfolg }(\mathrm{n}) \\
\text { Zeit (Mittel, SD) }\end{array}$ & $\begin{array}{c}\text { Erfolg (n) } \\
\text { Zeit (Mittel, SD) }\end{array}$ \\
\hline $1, \mathrm{n}=30$ & 26 & 30 \\
& $47,3 \pm$ & $34,8 \pm 13,5$ \\
\hline $2, \mathrm{n}=30$ & 24 & 29 \\
& $42,0 \pm 16,9$ & $35,7 \pm 13,6$ \\
\hline $3, \mathrm{n}=30$ & 27 & 29 \\
& $43,6 \pm 15,9$ & $36,3 \pm 13,5$ \\
\hline Gesamt, $\mathrm{n}=90$ & 77 & $88^{* a}$ \\
& $44,3 \pm 16,1$ & $35,6 \pm 13,5^{* * \mathrm{~b}}$ \\
\hline
\end{tabular}

\section{Tab. 3.2: Erfolgsraten und benötigte Zeit zur Ventilation}

Abkürzungen: BM-V, Ventilation über die Gesichtsmaske; ILMA-V, Ventilation über die Intubations-Larynxmaske; *a , $p<0,05$ (Wilcoxon-Test); **b , $p<0,001$ (rm ANOVA).

Die ILMA-V war bei einem Patienten nicht möglich, da nach Einleitung der Narkose die Mundöffnung unzureichend war und die ILMA nicht eingeführt werden konnte. Die Ventilation erfolgte bei diesem Patienten mit der Gesichtsmaske. Die 
laryngoskopische Intubation war nach Verabreichung des Muskelrelaxans problemlos möglich, da sich die Mundöffnung durch die neuromuskuläre Blockade deutlich verbesserte. Bei einem weiteren Patienten konnte die ILMA-V nicht zufriedenstellend durchgeführt werden, da es zu Undichtigkeiten der ILMA kam und die Kapnographie keine Plateau-Phase zeigte. Die ILMA-TI konnte bei diesem Patienten jedoch unproblematisch im ersten Versuch durchgeführt werden.

Die unzureichende Gesichtsmaskenbeatmung war in allen Fällen auf einen insuffizienten Schluss der Beatmungsmaske zurückzuführen, sodass eine kapnographisch dargestellte Plateau-Phase des endexspiratorischen $\mathrm{CO}_{2}$ nicht erreicht wurde. Bei all diesen Patienten war jedoch die Gesichtsmaskenbeatmung durch den Instruktor problemlos möglich, und die Probanden konnten mit der laryngoskopisch durchgeführten endotrachealen Intubation fortfahren.

Die mit Hilfe der Kaplan-Meier-Kurven erstellten mittleren Zeiten für eine erfolgreiche Ventilation war für die BM-V länger (45s, interquartile range, $30-60 \mathrm{~s}$ ), als für die ILMA-V (35s, interquartile range, $25-43 s, x^{2}-$ Test 20,8; $\left.p<0,01\right)$. 


\section{Ventilation}

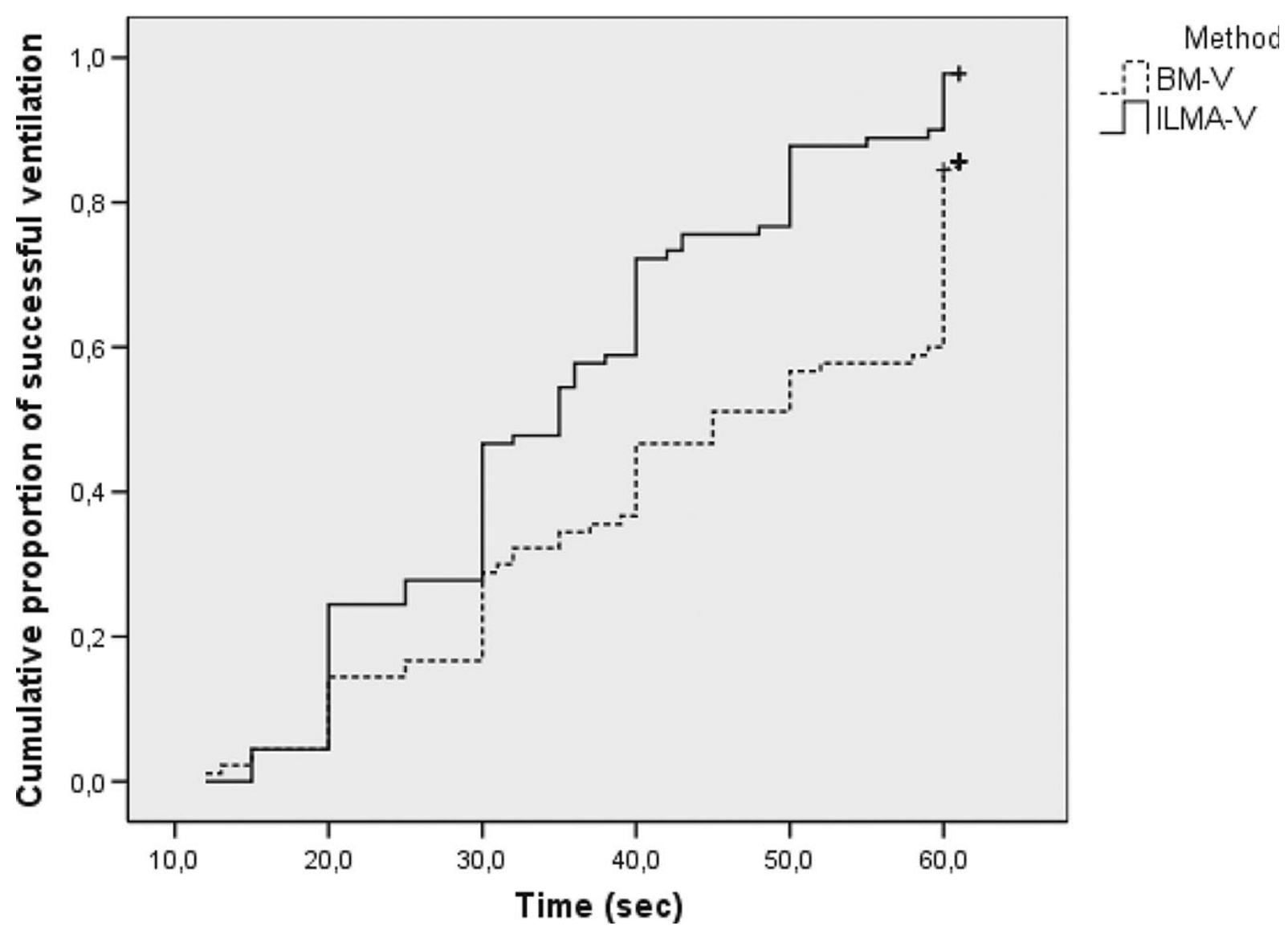

Abb. 3.1: Kaplan- Meier-Analyse für die Ventilation

Zeit in Sekunden, um eine erfolgreiche Ventilation zu etablieren. Vergleich der BM-V mit der ILMA-V (Kaplan-Meier-Analyse).

\subsubsection{Intubationsmaßnahmen}

Die endotracheale Intubation via ILMA war erfolgreicher als die LG-TI. So wurden signifikant weniger Intubationsversuche benötigt bei einer höheren Erfolgsrate über alle Versuchsdurchgänge (92,2\%), als bei der LG-TI $(60,0 \%$, p <0,001). Im ersten Versuch konnten mit der ILMA 75 Patienten (83\%) intubiert werden und weitere acht Patienten (9\%) im zweiten Versuch. Im Vergleich war die laryngoskopische Intubation im ersten Versuch bei $42 \%$ der Patienten und im zweiten Versuch bei 18\% der Patienten erfolgreich (38 bzw. 16 Patienten). Die Gesamtzeit bis zur Sicherung der Atemwege durch die Intubation war für die LG-TI signifikant 
länger, als für die ILMA-Intubation $(89,1 \pm 34,9$ s versus $45,7 \pm 29,1 \mathrm{~s}, F=99,6, p<0,01)$. Es konnte kein Einfluss bezüglich des Messzeitpunktes ( $F=0,1, p=0,90)$ noch bezüglich der Interaktion oder der angewandten Methode $(F=2,3, p>0,1)$ festgestellt werden (s. Tab. 3.3).

\begin{tabular}{|lcc|}
\hline & LG-TI & ILMA-TI \\
\cline { 2 - 3 } $\begin{array}{l}\text { Durchgang, } \\
\text { Anzahl der Patienten }\end{array}$ & $\begin{array}{c}\text { Erfolg }(\mathrm{n}) \\
\text { Zeit (Mittel, SD) }\end{array}$ & $\begin{array}{c}\text { Erfolg (n) } \\
\text { Zeit (Mittel, SD) }\end{array}$ \\
\hline $1, \mathrm{n}=30$ & 16 & 29 \\
& $94,7 \pm 33,4$ & $41,2 \pm 29,3$ \\
\hline $2, \mathrm{n}=30$ & 18 & 26 \\
$3, \mathrm{n}=30$ & $90,6 \pm 34,6$ & $46,5 \pm 33,2$ \\
& 20 & 28 \\
Gesamt, $\mathrm{n}=90$ & $82,1 \pm 36,4$ & $49,3 \pm 27,5$ \\
& 54 & $83^{* * a}$ \\
\hline
\end{tabular}

Tab. 3.3: Erfolgsraten und benötigte Zeit zur Intubation

Abkürzungen: LG-TI, laryngoskopisch durchgeführte endotracheale Intubation; ILMA-TI, Intubation via Intubationslarynxmaske; ${ }^{* *} a, p<0,05$ (Wilcoxon-Test); ${ }^{* *} b, p<0,001$ (rm ANOVA)

Drei von insgesamt 36 Patienten, bei denen die LG-TI primär nicht erfolgreich war, und zwei von sieben Patienten, bei denen die ILMA-TI primär nicht möglich war, wurden aus der zweiten Studienphase ausgeschlossen, da es zu leichten Erosionen an den Schleimhäuten der Atemwege gekommen war (Blutspuren auf dem Laryngoskop oder der ILMA). Von den verbleibenden 33 Patienten, bei denen die LG-TI im ersten Versuch nicht möglich war, konnten 28 (85\%) Patienten via ILMA erfolgreich intubiert werden. Im Vergleich konnte bei einem von insgesamt fünf Patienten, bei denen die ILMA-Insertion primär nicht möglich war, laryngoskopisch intubiert werden. War die ILMA-TI erfolglos, so konnte in keinem Fall die laryngoskopische Intubation durchgeführt werden. 
Die mit Hilfe der Kaplan-Meier-Kurven erstellten mittleren Zeiten für eine erfolgreiche Intubation war für die LG-TI länger (106s, interquartile range, 58 bis > $120 \mathrm{~s}$ ), als für die ILMA-TI (40s, interquartile range, $25-60$ s, $x^{2}-$ Test 58,3; P < $0,01)$.

\section{Tracheal intubation}

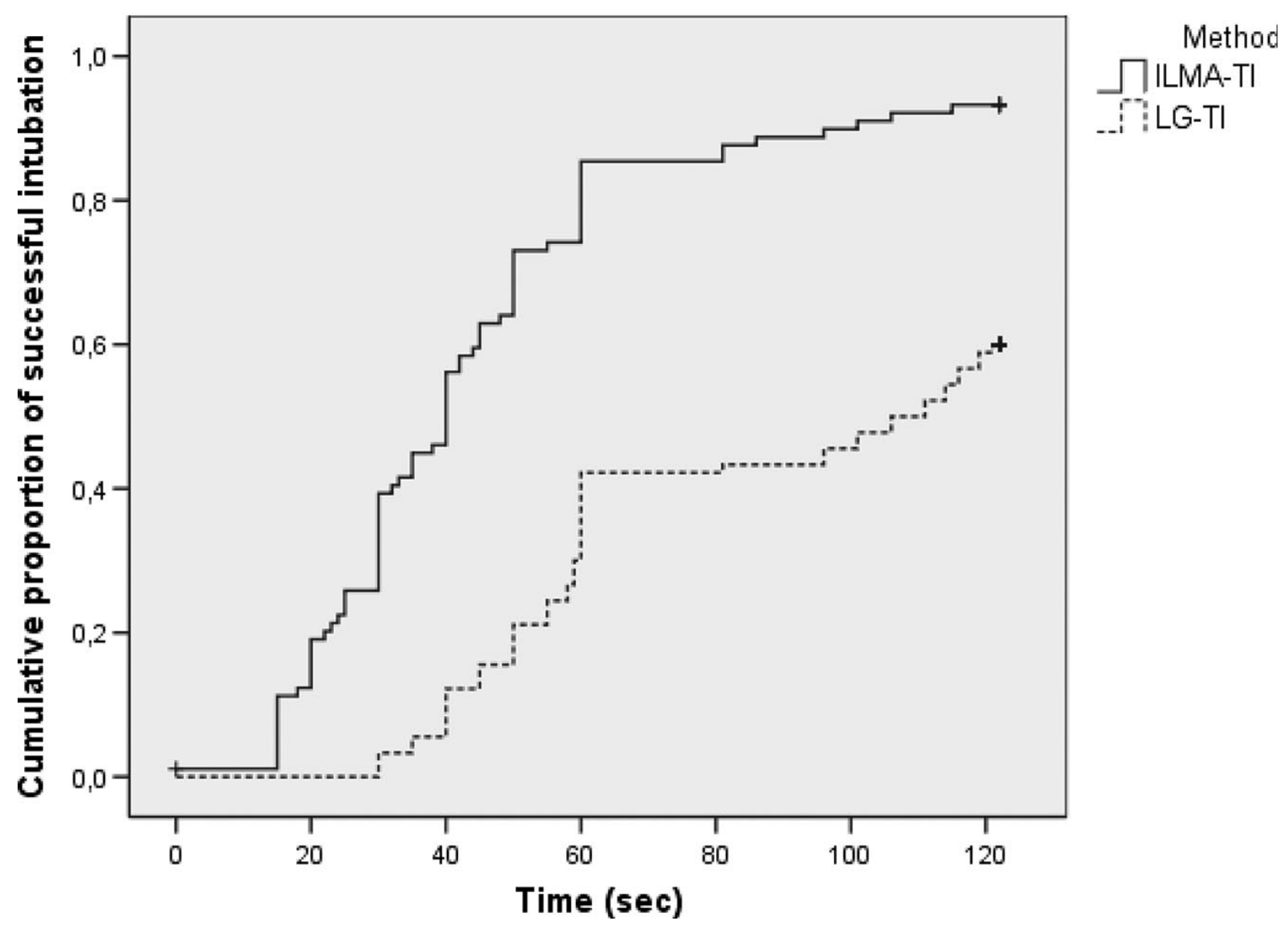

Abb. 3.2: Kaplan- Meier-Analyse für die Intubation

Zeit in Sekunden, um eine erfolgreiche Intubation zu etablieren. Vergleich der LG-TI mit der ILMA-TI (Kaplan-Meier-Analyse).

\subsubsection{Atemwegs-Hilfen und -Manöver}

Die Probanden konnten, wie oben beschrieben, Hilfsmittel und unterstützende Maßnahmen zur Ventilation oder Intubation anfordern. Es erfolgten für jede Atemwegssicherungsmaßnahme drei Durchgänge, sodass eine Gesamtzahl von 90 für jede Maßnahme resultierte. Für die BM-V wurde insgesamt 57 mal $(63,3 \%)$ von 
den Probanden der Güdel-Tubus, und 71 mal (78,9\%) von den Probanden die ZweiHand-Ventilation zur Unterstützung angefordert. Im Vergleich dazu, wurde nur 3 mal $(3,3 \%)$ von den Probanden die wiederholte Insertion der ILMA zur Verbesserung der Ventilation durchgeführt. Zur Verbesserung der Intubationsbedingungen wurde für die LG-TI 44 mal (48,9\%) das BURP-Manöver eingesetzt. Das Up-and-downManöver wurde zur Verbesserung der ILMA-TI hingegen nur 6 mal $(6,7 \%)$, das Chandi-Manöver 41 mal (46,1\%) von den Probanden angewandt (s. Tab. 3.4).

\section{Anzahl der Versuchsdurchgänge, n (\%)}

BM-V $(\mathrm{n}=90)$

Güdel- Tubus

Zwei- Hand- Ventilation

LG-TI $(\mathrm{n}=90)$

BURP-Manöver

ILMA-V ( $\mathrm{n}=90)$

Reinsertion

ILMA-TI $(\mathrm{n}=90)$

„Up-and-down"-Manöver

"Chandi"-Manöver
$57(63,3 \%)$

$71(78,9 \%)$

$44(48,9 \%)$

Tab. 3.4: Verwendete Atemwegs-Hilfen und -Manöver

Abkürzungen: BM-V, Ventilation via Gesichtsmaske; ILMA-V, Ventilation via Intubationslarynxmaske; LG-TI, laryngoskopisch durchgeführte endotracheale Intubation; ILMA-TI, Intubation via Intubationslarynxmaske; BURP-Manöver, backwards upwards right pressure (Druck auf den Schildknorpel). 


\section{$4 \quad$ Diskussion}

Die Sicherung der Atemwege ist eine fundamentale Maßnahme in der Behandlung kritisch kranker oder traumatisierter Patienten. Der Vorteil der endotrachealen Intubation im Vergleich zur Maskenbeatmung oder zur extraglottischen Atemwegssicherung ist, dass neben der Möglichkeit der Ventilation auch die Atemwege offen gehalten werden und eine Aspiration verhindert werden kann. Des Weiteren können adäquate Tidalvolumina während der Thoraxkompression bei kardiopulmonaler Reanimation und bei erhöhten Atemwegsdrücken verabreicht werden. Vorhandenes Sekret kann abgesaugt werden, und die Medikamentenapplikation ist endotracheal möglich (Nolan et al. 2005; Nolan et al. 2010; Timmermann 2009).

Die korrekt durchgeführte Maskenbeatmung ist eine essentiell wichtige Maßnahme während der kardiopulmonalen Reanimation, und soll einen hohen Stellenwert beim Reanimationstraining einnehmen (Nolan et al. 2005; Nolan et al. 2010). Die vorliegende Studie zeigt, dass $85 \%$ der Patienten erfolgreich mit der Gesichtsmaske beatmet werden können. Die dafür benötigte Zeit beträgt im Mittel 44 Sekunden. Im Gegensatz dazu können 98\% der Patienten mit der ILMA suffizient ventiliert werden. Die dafür benötigte Zeit beträgt im Mittel 36 Sekunden. Derzeit ist lediglich eine Studie von Avidan et al. publiziert, die die BM-V und LG-TI bei unerfahrenen Probanden mit der ILMA-V und ILMA-TI vergleichen (Avidan et al. 1999). Die BM-V konnte bei $72 \%$ und die ILMA-V konnte bei $98 \%$ der Teilnehmer erfolgreich durchgeführt werden. Andere Autoren beschreiben eine erfolgreiche BM-V von $43 \%$ verglichen mit einer Ventilationsrate von $80 \%$ bei anderen extraglottischen Atemwegshilfen für unerfahrene Probanden (Alexander et al. 1993; Clayton et al. 2001). Für die Gesichtsmaskenbeatmung wird mindestens eine Person benötigt. Die vorliegende Studie zeigt, dass $80 \%$ der Probanden eine ZweihandBeatmung zur suffizienten Ventilation benötigen, dies erfordert eine weitere Person zur Sicherstellung der Ventilation. Im Vergleich dazu kann die ILMA nach erfolgreicher Platzierung an ein Beatmungsgerät angeschlossen werden, sodass der personelle Aufwand geringer ist. Diese Situation kann im außerklinischen Bereich von Bedeutung sein, wenn die personellen Ressourcen begrenzt sind und mehrere Tätigkeiten parallel durchgeführt werden müssen. 
Die ERC-Richtlinien zur kardiopulmonalen Reanimation empfehlen die endotracheale Intubation als die anzustrebende Technik zur Atemwegssicherung. Diese Maßnahme soll nach den Richtlinien jedoch nur durchgeführt werden, wenn der Anwender ausreichende Erfahrung in dieser Technik aufweisen kann (Nolan et al. 2010). Die Ergebnisse der vorliegenden Studie zeigen eine Erfolgsrate der LG-TI von $60 \%$ für ungeübte Personen, und sind vergleichbar mit anderen Untersuchungen, die Erfolgsraten von 35-69\% bei Patienten bei elektiven Eingriffen im OP ermittelten (Davies et al. 1990; Pennant und Walker 1992; Reinhart und Simmons 1994; Bradley et al. 1998; Sayre et al. 1998; Avidan et al. 1999; Hohlrieder et al. 2006). Die LG-TI muss mindestens 50 bis 60 mal durchgeführt werden, bevor sie ohne größere Hilfestellungen bei Patienten mit normalen Atemwegsverhältnissen und unter elektiven Voraussetzungen mit optimalen Bedingungen im OP gelingt (Konrad et al. 1998; Mulcaster et al. 2003). Selbst diese Anforderungen sind jedoch schwierig für das gesamte medizinische Personal zu erfüllen, das in Notfallsituationen den Atemweg sichern muss.

In der vorliegenden Studie gelang die Intubation über die ILMA bei 92\% der Patienten signifikant häufiger erfolgreich und schneller als die LG-TI. Avidan et al. konnten 1999 aber hinsichtlich der ILMA-TI nur eine 43-prozentige Erfolgsrate erreichen. Jedoch waren in dieser Studie die Einlage- und Intubationstechnik unterschiedlich und die Anzahl der Anwendungen pro Teilnehmer nicht standardisiert (Avidan et al. 1999). Die Probanden unserer Studie mussten strikt die von Brimacombe beschriebene Einlagetechnik einhalten (Brimacombe et al. 1997). Weiterhin wurde das Chandi-Manöver bei $46 \%$ der durchgeführten Intubationen verwendet. Ferson et al. beschrieben im Jahr 2001 eine Verbesserung der Intubationsrate von 68\% auf 95\% unter Verwendung des Chandi-Manövers bei Patienten mit schwierigen Atemwegsverhältnissen (Ferson et al. 2001). Eine weitere Studie aus dem Jahre 2000 beschrieb in Analogie zu unseren Daten eine 86prozentige Erfolgsrate der ILMA-TI, wenn ungeübte Anwender diese durchführten (Doerges et al. 2000).

Für die Versorgung von Notfallpatienten ist neben der Erfolgsrate der endotrachealen Intubation auch die Zeitdauer der Intubation von entscheidender Bedeutung, insbesondere während der Durchführung einer CPR, wenn die Herzdruckmassage unterbrochen werden muss. Die Intubationszeiten waren in dieser Studie mit der ILMA signifikant kürzer, als mit der LG-TI (46 vs. 89 Sekunden). 
Aktuell liegt keine vergleichbare Literatur zu mittleren Intubationszeiten mit Hilfe der ILMA vor. Für die Einlage der klassischen LMA werden mittlere Zeiten von 23 bis 62 Sekunden angegeben (Tolley et al. 1992; Alexander et al. 1993; Burgoyne und Cyna 2001; Kurola et al. 2006). Für die LG-TI werden in vergleichbaren Studien mittlere Zeiten zwischen 88 und 206 Sekunden beschrieben (Pennant und Walker 1992; Reinhart und Simmons 1994; Hohlrieder et al. 2006).

In Analogie zur konventionellen Larynxmaske kann auch für die ILMA ein verbesserter Aspirationsschutz während der CPR im Vergleich zur BM-V angenommen werden (Reeves et al. 2004). Die ILMA-Ventilation muss während und zwischen den Intubationsversuchen nicht unterbrochen werden, wenn ein Beatmungsgerät mit einem entsprechenden Adapter angeschlossen wird. Alternativ zur sofortigen ILMA-TI kann die Intubation auch auf einen späteren Zeitpunkt verschoben werden, z.B. nach Wiedererlangen des Kreislaufes oder nach Ankunft im Zielkrankenhaus, wenn mehr technische und personelle Ressourcen zur Verfügung stehen.

Die besondere Stärke der ILMA liegt im Management des schwierigen Atemweges. Die vorliegende Studie konnte zeigen, dass 75\% (28 von 33) der Patienten, bei denen primär die LG-TI scheiterte, mit der ILMA-TI intubiert werden konnten. Die LG-TI nach gescheiterter ILMA-TI gelang jedoch nur bei einem Patienten, bei dem sich erst nach vollständiger neuromuskulärer Blockade die Mundöffnung wieder normalisierte. Die ILMA-TI durfte bei diesem Patienten aufgrund des Studienprotokolls nicht durchgeführt werden. Die hohe Erfolgsrate der ILMA-TI nach gescheiterter LG-TI verdeutlicht den Stellenwert dieses Instrumentes auch für den ungeübten Anwender, denn alle Intubationen wurden unter kontrollierten Bedingungen im OP bei Patienten mit elektiven Eingriffen ohne Hinweise auf schwierige Atemwegsverhältnisse durchgeführt. Im Gegensatz dazu muss die Intubation unter Notfallbedingungen als deutlich schwieriger eingeschätzt werden (Rosenblatt und Murphy 1999). Gerade in präklinischen Situationen können durch Sekrete und Blutungen, subkutane Emphyseme, Verletzungen der oberen Atemwege oder die Immobilisation der Halswirbelsäule die Maskenbeatmung oder die Laryngoskopie erschwert sein (Stone et al. 1998). Die schwierige Atemwegssicherung tritt in der Regel unerwartet auf, häufig noch verstärkt durch respiratorische Insuffizienz mit Hypoxie und limitiertem Zugang zum Kopf des Patienten. Weitere Faktoren, die das Atemwegsmanagement erschweren, liegen in 
gleichzeitig durchzuführenden Maßnahmen, unterschiedlichen Graden der Bewusstseinsänderungen und dem Fehlen einer neuromuskulären Blockade. In diesem Zusammenhang ermittelten Rosenblatt und Murphy, dass im Vergleich zum Atemwegsmanagement innerhalb der Klinik das präklinische Atemwegsmanagement eine erhöhte Inzidenz der schwierigen und erfolglosen LG-TI sowie eine schlechtere Sicht auf die Stimmbänder zeigte, wenn erfahrene Notärzte aus dem Bereich der Anästhesiologie die LG-TI durchführten (Rosenblatt und Murphy 1999). Des Weiteren steigt die Zahl von Komplikationen und unerwünschten Ereignissen mit der Anzahl der Intubationsversuche. So konnte Mort (Mort 2004) zeigen, dass mit steigender Anzahl der Intubationsversuche auch die Rate an Komplikationen, als da wären Hypoxie, Regurgitation, Aspiration oder kardiale Nebenwirkungen, ansteigt.

Der Goldstandard der Atemwegssicherung ist die endotracheale Intubation. Je nach Qualifikation des Helfers kann es jedoch geboten sein, ein Ersatzverfahren der Atemwegssicherung zu benutzen (Nolan et al. 2010). Es stellt sich nun die Frage, welche der supraglottischen Atemwegshilfen zur Anwendung kommen sollten. Als Ersatzverfahren kommen supraglottische Atemwege (SGA) z.B. die Larynxmaske, der Larynxtubus oder der Combitubus in Frage. Alle SGA scheinen zwei gemeinsame Vorteile gegenüber der konventionellen Atemwegssicherung zu haben. Zum einen ist die Ventilation effektiver, schneller und sicherer als bei der Maskenbeatmung, zum anderen erfolgt die Einlage durch unerfahrenes Personal schneller und erfolgreicher als die Durchführung einer laryngoskopisch vermittelten endotrachealen Intubation.

Larynxmasken (LMA) sind für das Management des schwierigen Atemweges im OP nahezu von allen anästhesiologischen Fachgesellschaften empfohlen (Henderson et al. 2004; Braun et al. 2004). Der Grund für diese Empfehlung liegt darin begründet, dass eine schwierige Maskenbeatmung oder eine schwierige laryngoskopische Intubation keine Limitierung einer LMA-Anwendung darstellt. Larynxmasken werden in der täglichen Routine häufig angewendet, aus diesem Grund besteht beim Benutzer dieses Atemwegshilfsmittels eine große Anwendungssicherheit. Die Vorteile der LMA im präklinischen Einsatz und unter Reanimationsbedingungen sind das schnelle und leichte Einführen, die rasche Erlernbarkeit, die Vermeidung einer ösophagealen Intubation und ihre Anwendung bei Patienten mit Atemwegsanomalien. Die LMA ist jedoch nicht geeignet für die Verabreichung hoher Atemwegsdrücke oder nach pulmonaler Aspiration (Keller und 
Brimacombe 2007). Eine endotracheale Intubation ist über die liegende klassische LMA jedoch nicht möglich. Bei Anwendung der ILMA kann im Verlauf oder primär eine endotracheale Intubation erfolgen. Bei nahezu identischem Vorgehen kann mit der ILMA ein Aspirationsschutz erreicht werden, bei der klassischen LMA ist dies nicht der Fall.

Im Bemühen um eine einfache Alternative (Katz und Falk 2001) zur Sicherung der Atemwege und zur adäquaten Beatmung im Notfall wurde der Combitubus ${ }^{\mathrm{TM}}$ entwickelt. Es handelt sich bei diesem Instrument um einen ösophagealen Verschlusstubus, der vor allem wegen seiner einfachen Anwendung und des potentiellen Schutzes vor Aspiration durch den ösophagealen Ballon Verwendung findet (Krafft und Schebesta 2004). Der Combitubus ${ }^{\mathrm{TM}}$ ist seit 1992 international etabliert (Baskett und Nolan 1996) und gilt in den meisten Notfallsystemen als eine Alternative zur Sicherung der Atemwege. Der entscheidende Nachteil ist jedoch, dass es bei Anwendung des Combitubus zur hohen Atemwegsmorbidität kommen kann, was die Ausbildung des notfallmedizinischen Personals an diesem Atemwegshilfsmittel doch deutlich erschwert. Obgleich es in der Literatur Berichte zur erfolgreichen Erstanwendung ohne jedwede Übung gibt (Enlund et al. 2001), werden das Training und eine regelmäßige Auffrischung der Kenntnisse zumindest am Intubationstrainer empfohlen, da eine routinemäßige Anwendung im OP bei Patienten mit elektiven Eingriffen wegen der hohen Atemwegsmorbidität kaum in Betracht kommt.

Eine Weiterentwicklung des ösophagealen Verschlusstubus ist der Larynxtubus $^{\mathrm{TM}}$ (VBM Medizintechnik $\mathrm{GmbH}$ ). Es handelt sich bei dem Larynxtubus ${ }^{\mathrm{TM}}$ um einen Silikontubus mit weicher Spitze und flexiblem Atemwegskanal, wodurch die Verletzungsgefahr vermindert wird. Der Larynxtubus ${ }^{\mathrm{TM}}$ ist mit zwei korrespondierenden Cuffs versehen, von denen der distale Cuff den Ösophagus verschließt, und der proximale Cuff oropharyngeal zu liegen kommt, um ein Entweichen der Luft nach oral zu verhindern. Einschränkend zu diesem Instrument muss allerdings erwähnt werden, dass dieses kaum im Routinebetrieb im OP Verwendung findet, und damit nur schwer zu Ausbildungszwecken zur Verfügung steht. Eine tracheale Lage des Larynxtubus ${ }^{T M}$ stellt eine Fehllage dar und führt zur kompletten Atemwegsobstruktion mit deletärem Ausgang. Die Summe der publizierten Ergebnisse kann keine Vorteile bei der Anwendung am Patienten im direkten Vergleich zur LMA zeigen (Kurola et al. 2006). 
Eine weitere Möglichkeit, die endotracheale Intubation durchzuführen, ist die Anwendung der videoassistierten Laryngoskopie. In den letzten Jahren finden verschiedene videoassistierte Techniken zur indirekten (z.B. GlideScope (Cooper 2003), Airtraq (Maharaj et al. 2006), McGrath Laryngoskopie (Shippey et al. 2007)) und direkten (z.B. C-Mac (Meininger et al. 2010)) Laryngoskopie Verbreitung. Die Innovation der Videolaryngoskopie besteht in der Einarbeitung einer Glasfaseroptik in den Standardlaryngoskopiespatel. Die optischen Fasern enden zusammen mit den Fasern der Lichtquelle im distalen Bereich des Spatels. Durch die Optik wird der Blickwinkel nach ventral vergrößert, was zu einer relevanten Verbesserung der Sichtverhälnisse führen kann. Hofstetter et al. (Hofstetter et al. 2006) konnten an einem Patientenkollektiv zeigen, dass eine Verbesserung der Sichtverhältnisse durch die Videolaryngoskopie erzielt werden konnte. Aufgenommen in diese Studie wurden Patienten mit elektiven Eingriffen ohne Anzeichen schwieriger Intubation. Demgegenüber konnten Meininger et al. (Meininger et al. 2010) an einem Patientenkollektiv mit erwartet hoher Rate an schwierigen Intubationen (Patienten, die sich einem HNO-ärztlichen Eingriff unterzogen) zeigen, dass die Sichtverhältnisse auf die Stimmbandebene im Vergleich zur konventionellen Laryngoskopie verbessert werden konnten. Die vorliegenden Ergebnisse lassen den Schluss zu, dass mit Hilfe der Videolaryngoskopie bessere Sichtverhältnisse auf Stimmbandebene erreicht werden können, und somit die endotracheale Intubation erleichtert werden kann. Ob diese Ergebnisse auf den notfallmedizinischen Bereich übertragen werden können und ob dieses auch für ungeübtes notfallmedizinisches Personal zutrifft, kann zum jetzigen Zeitpunkt nicht abschließend beurteilt werden.

Die Tatsache, dass der Vorgang der Laryngoskopie und Intubation neben dem Durchführenden auch von weiteren Personen direkt und ohne Sichtbehinderung beobachtet werden kann, eröffnet neue Perspektiven für die Ausbildung. Mit der Videolaryngoskopie kann die Technik der Laryngoskopie gezeigt und vermittelt werden, des Weiteren kann die korrekte Positionierung des Endotrachealtubus visuell verifiziert werden (Kaplan et al 2002; Weiss et al. 2001). Ob und inwieweit die Lernkurve zur korrekten Durchführung der direkten Laryngoskopie bzw. endotrachealen Intubation durch die Verwendung der Videolaryngoskopie optimiert 
werden kann, wird Gegenstand zukünftiger Untersuchungen sein (Hofstetter et al. 2006). 


\section{$5 \quad$ Zusammenfassung und Schlussfolgerung}

Das Atemwegsmanagement spielt eine zentrale Rolle in der Versorgung von Notfallpatienten. Da jedoch die Gesichtsmaskenbeatmung (BM-V) und die laryngoskopische Intubation (LG-TI) von ungeübten Personen häufig nicht erfolgreich durchgeführt werden können, wurde eine alternative Methode untersucht.

Dreißig Studierende der Humanmedizin im Praktischen Jahr ohne Erfahrung mit Maßnahmen des Atemwegsmanagements nahmen an einer prospektiv, randomisierten Crossover-Untersuchung teil. Verglichen wurden die Erfolgsraten und die Zeitdauer der BM-V und LG-TI mit der Ventilation und Intubation über die Intubations- Larynxmaske (ILMA-V / ILMA-TI). Nach einer theoretischen Einführung und der Übung am Intubationstrainer führte jeder Studierende je drei Atemwegssicherungen mittels BM-V und LG-TI sowie ILMA-V und ILMA-TI in randomisierter Reihenfolge bei Patienten im Operationssaal ohne Anzeichen einer schwierigen Intubation durch. Die Zeit für die Durchführung der Atemwegssicherungsmaßnahme wurde auf 60 Sekunden begrenzt. Konnte auch nach zwei Versuchen die Intubation nicht erfolgreich durchgeführt werden, wurde die jeweils alternative Methode angewendet.

Die ILMA-V konnte signifikant häufiger erfolgreich (97,8\% vs. 85,6\%, p<.05) und schneller $(35,6 \pm 8,0$ versus $44,3 \pm 10,8$ Sekunden, $p<.01)$ durchgeführt werden, als die BM-V. Die Intubation scheiterte signifikant häufiger $(40,0 \%$ vs. $7,8 \%, p<.01)$ und dauerte länger $(45,7 \pm 14,8$ vs. $89,1 \pm 23,3$ Sekunden, $p<.01)$ mit der LG-TI verglichen mit der ILMA-TI. Nach gescheiterter LG-TI konnte die ILMA-TI in 28 von 33 (85\%) Fällen erfolgreich durchgeführt werden, während dies nur in einem von fünf Fällen mit der LG-TI nach gescheiterter ILMA-TI gelang.

Unsere Ergebnisse unterstützen die Forderung nach einer Implementierung alternativer Verfahren zur Atemwegssicherung in das medizinische Curriculum und in die Ausbildung von notfallmedizinischem Personal. Für diejenigen Personen, die die laryngoskopische Intubation nicht regelmäßig durchführen, sollte eine prinzipielle Verwendung der ILMA zur Atemwegssicherung erwogen werden. 
Adnet F, Jouriles NJ, Le Toumelin P, Hennequin B, Taillandier C, Rayeh F, Couvreur J, Nougière B, Nadiras P, Ladka A, Fleury M (1998): Survey of out-of-hospital emergency intubations in the French prehospital medical system: a multicenter study. Ann Emerg Med 32(4): 454-60.

Ärztekammer Niedersachsen (2004): Weiterbildungsordnung der Ärztekammer Niedersachsen vom 27.11.2004, Abschnitt A §5

Ärztekammer Niedersachsen 2004: Abschnitt C Abs. 31 S.160 Weiterbildungsordnung der Ärztekammer Niedersachsen vom 27.11.2004

Alexander R, Hodgson P, Lomax D, Bullen C (1993): A comparison of the laryngeal mask airway and Guedel airway, bag and facemask for manual ventilation following formal training. Anaesthesia 48(3), 231-234

Avidan MS, Harvey A, Chitkara N, Ponte J (1999): The intubating laryngeal mask airway compared with direct laryngoscopy. Br J Anaesth $\underline{83(4)}, 615-617$

Baskett PJ (1994): The use of the laryngeal mask airway by nurses during cardiopulmonary resuscitation._Anaesthesia $\underline{49}, 3-7$

Baskett PJ, Nolan J, Parr M (1996): Tidal volumes which are perceived to be adequate for resuscitation. Resuscitation $\underline{31(3)}, 231-234$

Bellhouse CP, Dore C (1988): Criteria for estimating likelihood of difficulty of endotracheal intubation with the Macintosh laryngoscope. Anaesth Intensive Care 16(3), 329-337

Bowman FP, Menegazzi JJ, Check BD, Duckett TM (1995): Lower esophageal sphincter pressure during prolonged cardiac arrest and resuscitation. Ann Emerg Med 26(2), 216-219

Bradley JS, Billows GL, Olinger ML, Boha SP, Cordell WH, Nelson DR (1998): Prehospital oral endotracheal intubation by rural basic emergency medical technicians. Ann Emerg Med 32(1), 26-32

Brain AIJ (1983): The laryngeal mask - a new concept in airway management. $\mathrm{Br} \mathrm{J}$ Anaesth 55(8), 801-805

Brain AIJ, Verghese C, Addi EV, Kapila A (1997a): The intubating laryngeal mask. I: Development of a new device for intubation of the trachea. $\mathrm{Br} \mathrm{J}$ Anaesth $\underline{79(6)}$, 699-703

Brain AIJ, Verghese C, Addi EV, Kapila A, Brimacombe JR (1997b): The intubating laryngeal mask. II: A preliminary clinical report of a new means of intubating the trachea. Br J Anaesth 79(6), 704-709

Braun U, Goldmann K, Hempel V, Krier C (2004): Airway Management. Leitlinien der Deutschen Gesellschaft für Anästhesiologie und Intensivmedizin. Anaesth Intensivmed $\underline{45}$, 302-306

Brimacombe JR: Intubating LMA for Airway intubation; in: Laryngeal mask anesthesia; hrsg. v. Saunders Verlag, Philadelphia 2005, 469-504

Brimacombe JR, Brain AIJ, Berry A: The laryngeal mask airway: A review and practice guide; in: Laryngeal Mask Airway (LMA); hrsg. v. Brimacombe, JR, Saunders Verlag, Phildelphia 1997, 216 - 277

Bulger EM, Copass MK, Maier RV, Larsen J, Knowles J, Jurkovich J (2002): An analysis of advanced prehospital airway management. J Emerg Med 23(2), 183-189

Burgoyne LL, Cyna AM (2001): Laryngeal mask vs intubating laryngeal mask: insertion and ventilation by inexperienced resuscitators. Anaesth Intensive Care 29(6), 604-608 
Choyce A, Avidan MS, Patel C, Harvey A, Timberlake C, McNeilis N, Glucksman E (2000): Comparison of laryngeal mask and intubating laryngeal mask insertion by the naive intubator. Br J Anaesth 84(1), 103-105

Choyce A, Avidan MS, Shariff A, Del Aguila M, Redcliffe JJ, Chan T (2001): A comparison of the intubating and standard laryngeal mask airways for airway management by inexperienced personnel. Anaesthesia 56(4), 357-360

Clayton TJ, Pittman JA, Gabbott DA (2001): A comparison of two techniques for manual ventilation of the lungs by non-anaesthetists: the bag-valve-facemask and the cuffed oropharyngeal airway (COPA) apparatus. Anaesthesia 56(8), 756-759

Cook TM, Hommers C (2006): New airways for resuscitation? Resuscitation $\underline{69(3)}$, 371-387

Cooper RM (2003): Use of a new videolaryngoscope (GlideScope) in the management of a difficult airway. Can J Anaesth $\underline{50}, 611-613$

Davies PRF, Tighe SQM, Greenslade GL, Evans GH (1990): Laryngeal mask airway and tracheal tube insertion by unskilled personnel. Lancet 336(8721), 977-979

Dirks B: Sicherung der Atemwege in der Notfallmedizin; in: Airway-Management - Die Sicherung der Atemwege; hrsg. v. Krier C, Georgi R; Thieme Verlag, Stuttgart - New York 2001, 297-303

Doerges V (2005): Airway management in emergency situations. Best Pract Res Clin Anaesthesiol 19(4), 699-715

Doerges V, Wenzel V (2001): Ventilation zur kardiopulmonalen Reanimation. Intensivmedizin $\underline{38}$, 576-589

Doerges V, Sauer C, Ocker H, Wenzel V, Schmucker P (1999a): Airway management during cardiopulmonary resuscitation - a comparative study of bag-valve-mask, laryngeal mask airway and combitube in a bench model. Resuscitation 41(1), 63-69

Doerges V, Sauer C, Ocker H, Wenzel V, Schmucker P (1999b): Smaller tidal volumes during cardiopulmonary resuscitation: comparison of adult and paediatric self-inflatable bags with three different ventilatory devices. Resuscitation 43(1), 31-37

Doerges V, Wenzel V, Neubert E, Schmucker P (2000): Emergency airway management by intensive care unit nurses with the intubating laryngeal mask airway and the laryngeal tube. Crit Care 4(6) 369-376

Doerges V, Wenzel V, Schumann T, Neubert E, Ocker H, Gerlach K (2001): Intubating laryngeal mask airway, laryngeal tube, $1100 \mathrm{ml}$ self-inflating bagalternatives for basic life support?. Resuscitation 51(2), 185-191

Enlund M, Miregard M, Wennmalm K (2001): The Combitube for failed intubation instructions for use. Acta Anaesthesiol Scand 45(1), 127-128

Ferson DZ, Rosenblatt WH, Johansen MJ Osborn I (2001): Use of the intubating LMA-Fastrach in 254 patients with difficult-to-manage airways. Anesthesiology 95(5), 1175-1181

Gabrielli A, Wenzel V, Layon AJ, von Goedecke A, Verne NG, Idris AH (2005): Lower esophageal sphincter pressure measurement during cardiac arrest in humans: potential implications for ventilation of the unprotected airway. Anesthesiology 103(4), 897-899

Gausche M, Lewis RJ, Stratton SJ, Haynes BE, Gunter CS, Goodrich SM, Poore PD, McCollough MD, Henderson DP, Pratt FD, Seidel JS (2000): Effect of out-ofhospital pediatric endotracheal intubation on survival and neurological outcome: a controlled clinical trial. Jama 283(6), 783-790 
Grayling M, Wilson IH, Thomas B (2002): The use of the laryngeal mask airway and combitube in cardiopulmonary resuscitation; a national survey. Resuscitation 52(2), 183-186

Henderson JJ, Popat MT, Latto IP, Pearce AC (2004): Difficult Airway Society guidelines for management of the unanticipated difficult intubation. Anaesthesia $\underline{59}, 675-694$

Hofstetter C, Scheller B, Flondor M, Gerig HJ, Heidegger T, Brambrink A, Thierbach A, Wilhelm W, Wrobel M, Zwißler B (2006): Videolaryngoskopie versus direkte Laryngoskopie zur elektiven endotrachealen Intubation. Anaesthesist 55(5), 535-540

Hohlrieder M, Brimacombe JR, von Goedecke A, Keller C (2006): Guided insertion of the ProSeal laryngeal mask airway is superior to conventional tracheal intubation by first-month anesthesia residents after brief manikin-only training. Anesth Analg 103(2), 458-462

Jemmett ME, Kendal KM, Fourre MW, Burton JH (2003): Unrecognized misplacement of endotracheal tubes in a mixed urban to rural emergency medical services setting. Acad Emerg Med 10(9), 961-965

Jones JH, Murphy MP, Dickson RL, Somerville JJ, Brizendine EJ (2004): Emergency physician-verified out-of-hospital intubation: miss rates by paramedics. Acad Emerg Med 11(6), 707-709

Kaplan MB, Ward DS, Berci G (2002): A new video laryngoscope - an aid to intubation and teaching. J Clin Anesth 14(8), 620-626

Katz SH, Falk JL (2001): Misplaced endotracheal tubes by paramedics in an urban emergency medical services system. Ann Emerg Med 37(1), 32-37

Keller C, Brimacombe JR: Larynxmaske; in: Management des schwierigen Atemwegs; hrsg. v. Doerges V, Paschen HR, Springer Verlag, Berlin 2007, 368-385

Knill RL (1993): Difficult laryngoscopy made easy with a "BURP". Can J Anaesth $\underline{40(3)}, 279-282$

Konrad C, Schüpfer G, Wietlisbach M, Gerber H (1998): Learning manual skills in anesthesiology: Is there a recommended number of cases for anesthetic procedures?. Anesth Analg 86(3), 635-639

Krafft P, Schebesta K (2004): Alternative management techniques for the difficult airway: esophageal-tracheal Combitube. Curr Opin Anaesthesiol 17(6), 499504

Kurola J, Pere $P$, Niemi-Murola L, Silfvast $T$, Kairaluoma $P$, Rautoma $P$, Castrèn $M$ (2006): Comparison of airway management with the intubating laryngeal mask, laryngeal tube and CobraPLA $^{\circledR}$ by paramedical students in anaesthetized patients. Acta Anaesthesiol Scand 50(1), 40-44

Langenstein H, Möller F (1998): Erste Erfahrungen mit der Intubationslarynxmaske. Anaesthesist 47(4), 311-319

Langeron O, Masso E, Huraux C, Guggiari M, Bianchi A, Coriat P, Riou B (2000): Prediction of difficult mask ventilation. Anesthesiology 92(5), 1229-1236

Latto IP, Vaughan RS: Difficultys in tracheal intubation, 2nd Edition; Saunders Verlag, London 1997

Lawes EG, Baskett PJF (1987): Pulmonary aspiration during unsuccessful cardiopulmonary resuscitation. Intensive Care Med 13(6), 379-382

LMA- Fastrach ${ }^{\mathrm{TM}}$ Anleitungshandbuch, LMA, Laryngeal Mask Company, Hemsley on the Thames, Großbritannien 2002, 8-11

Maharaj CH, O'Croinin D, Curley G, Harte BH, Laffey JG (2006): A comparision of tracheal intubation using the Airtraq or the Macintosh laryngoscope in routine 
airway management: a randomised, controlled clinical trail. Anaestesia $\underline{61}$, 1093-1099

Martin SE, Ochsner MG, Jarman RH, Agudelo WE, Davis FE (1999): Use of the laryngeal mask airway in air transport when intubation fails. J Trauma $\underline{47(2)}$, 352-357

Meininger D, Struhal U, Weber CF, Fogl D, Holzer L, Zacharowski K, Byhahn C (2010): Direkte Lanrygokopie oder C-MAC Videolaryngoskopie? Routineintubation von Patienten in der HNO-Heilkunde. Anaesthesist 59(9), 806-811

Mort TC (2004): Emergency tracheal intubation: complications associated with repeated laryngoscopic attempts. Anaesth Analg 99(2), 607-613

Morton HJ, Wylie WD (1951): Anaesthetic deaths due to regurgitation or vomiting. Anaesthesia 6(4), 190-201

Mulcaster JT, Mills J, Hung OR, MacQuarrie K, Law JA, Pytka S, Imrie D, Field C (2003): Laryngoscopic intubation: learning and performance. Anesthesiology 98(1), 23-27

Niedersächsisches Rettungsdienstgesetz (NRettDG) §10 Abs.1 vom 02.10.2007 (Nds. GVBI. Nr.31/2007 S.473)

Nolan JP (2001): Prehospital and resuscitative airway care: should the gold standard be reassessed?. Curr Opin Crit Care 7(6), 413-421

Nolan JP, Deakin CD, Soar J, Böttiger BW, Smith G (2005): European Resuscitation Council guidelines for resuscitation 2005. Section 4. Adult advanced life support. Resuscitation 67 Suppl 1, 39-86

Nolan JP, Soar J, Zideman DA, Biarent D, Bossaert LL, Deakin C, Koster RW, Wyllie J, Böttiger B (2010): European Resuscitation Council guidelines for resuscitation 2010. Section 1. Executive summary. Resuscitation 81(10), 1219-1276

Patil VU, Stehling LC, Zauder HL (1983): Prediction the difficulty of intubation utilizing an intubation gauge. Anaesthesiol Rev 10, 31-33

Pennant JH, Walker MB (1992): Comparison of the endotracheal tube and laryngeal mask in airway management by paramedical personnel. Anesth Analg $\underline{74(4)}$, 531-534

Reeves MD, Skinner MW, Ginifer CJ (2004): Evaluation of the Intubating Laryngeal Mask Airway used by occasional intubators in simulated trauma. Anaesth Intensive Care 32(1), 73-76.

Reinhart DJ, Simmons G (1994): Comparison of placement of the laryngeal mask airway with endotracheal tube by paramedics and respiratory therapists. Ann Emerg Med 24(2), 260-263

Ridgway S, Hodzovic I, Woollard M, Latto IP (2004): Prehospital airway management in Ambulance Services in the United Kingdom. Anaesthesia 59(11), 10911094

Roessler M, Zuzan O (2006): EMS systems in Germany. Resuscitation 68(1), 45-49

Rosenblatt WH, Murphy M (1999): The intubating laryngeal mask: use of a new ventilating-intubating device in the emergency department. Ann Emerg Med 33(2), 234-238

Ruben H, Knudsen EJ, Carugati G (1961): Gastric inflation in relation to airway pressure. Acta Anaesthesiol Scand $\underline{5}, 107-114$

Samsoon GLT, Young JRB (1987): Difficult tracheal intubation: a retrospective study. Anaesthesia 42(5), 487-490

Sasada MP, Gabbott DA (1994): The role of the laryngeal mask airway in prehospital care. Resuscitation 28(2), 97-102 
Savva D (1994): Prediction of difficult tracheal intubation. Br J Anaesth $\underline{73(2)}$, 149153

Sayre MR, Sakles JC, Mistler AF, Evans JL, Kramer AT, Pancioli AM (1998): Field trial of endotracheal intubation by basic EMTs. Ann Emerg Med 31(2), 228233

Shippey B, Ray D, McKeown D (2007): Case series: The MacGrath videolaryngoscope - An initial clinical evaluation. Can J Anaesth $\underline{54}$ 307-313

Silvestri S, Ralls GA, Krauss B, Thundiyil J, Rothrock SG, Senn A, Carter E, Falk J (2005). The effectiveness of out-of-hospital use of continuous end-tidal carbon dioxide monitoring on the rate of unrecognized misplaced intubation within a regional emergency medical services system. Ann Emerg Med 45(5), 497-503

Slagt C, Zondervan A, Patka P, de Lange JJ (2004): A retrospective analysis of the intubations performed during 5 years of helicopter emergency medical service in Amsterdam. Air Med J 23(5), 36-37

Spence AA, Moir DD, Finlay WEI (1967): Observations on intragastric pressure. Anaesthesia 22(2), 249-256

Stone BJ, Chantler PJ, Baskett PJF (1998): The incidence of regurgitation during cardiopulmonary resuscitation: a comparison between the bag valve mask and laryngeal mask airway. Resuscitation 38(1), 3-6

Thierbach A, Piepho T, Wolcke B, Küster S, Dick W (2004): Präklinische Sicherung der Atemwege. Anaesthesist 53(6), 543-550

Timmermann A (2009): Die endotracheale Intubation - ist der Goldstandard in Gefahr?. Notfall- und Rettungsmedizin 34, 47-54

Timmermann A, Eich C, Russo SG, Natge U, Bräuer A, Rosenblatt WH, Braun U (2006): Prehospital airway management: a prospective evaluation of anaesthesia trained emergency physicians. Resuscitation $\underline{70(2)}$, 179-185

Timmermann A, Russo SG, Eich C, Roessler M, Braun U, Rosenblatt WH, Quintel M (2007a): The out-of-hospital esophageal and endobronchial intubations performed by emergency physicians. Anesth Analg 104(3), 619-623

Timmermann A, Braun U, Panzer W, Schlaeger M, Schnitzker M, Graf BM (2007b): Präklinisches Atemwegsmanagement in Norddeutschland: Individuelle Kenntnisse, Vorgehen und Ausrüstung. Anaesthesist 56, 328-334

Tolley PM, Watts ADJ, Hickman JA (1992): Comparison of the use of the laryngeal mask and face mask by inexperienced personnel. Br J Anaesth 69(3), 320-321

Wang HE, Peitzman AB, Cassidy LD, Adelson PD, Yealy DM (2004): Out-of-hospital endotracheal intubation and outcome after traumatic brain injury. Ann Emerg Med 44(5), 439-450

Weiss M, Schwarz U, Dillier CM, Gerber AC (2001): Teaching and supervising tracheal intubation in paediatric patients using videolaryngoscopy. Pediatric Anesthesia 11(3), 343-348

Wenzel V, Idris AH, Banner M, Kubilis PS, Williams JL (1998): Influence of tidal volume on the distribution of gas between the lungs and stomach in the nonintubated patient receiving positive-pressure ventilation. Crit Care Med 26(2), 364-368

Wenzel V, Keller C, Idris AH, Dörges V, Lindner KH, Brimacombe JR (1999): Effects of smaller tidal volumes during basic life support ventilation in patients with respiratory arrest: good ventilation, less risk?. Resuscitation $\underline{43(1)}, 25-29$ 


\section{Danksagung}

Mit dem Abschluss der vorliegenden Dissertation möchte ich mich bei denen bedanken, die mir mit Rat und Tat zur Seite standen.

Mein ganz besonderer Dank gilt Herrn PD Dr. med. A. Timmermann für die Bereitstellung des Themas und die wohlwollende Begleitung und ausgezeichnete Unterstützung bei der Fertigstellung dieser Arbeit.

Mein Dank gilt Herrn Prof. Dr. med. U. Braun und Herrn Prof. Dr. med. T. Crozier für die konstruktiven Anregungen bei der Anfertigung dieser Dissertation.

Ferner danke ich dem Zentrum Anaesthesiologie, Rettungs- und Intensivmedizin für die Unterstützung während der Durchführung der gesamten Arbeit. 


\section{Lebenslauf}

Ich wurde am 12. Juni 1960 in Holzminden geboren. Von 1966 bis 1975 besuchte ich die Volksschule in Neuhaus im Solling sowie die Grund- und Hauptschule Am Grimmenstein in Holzminden, und von 1975 bis 1977 die Berufsfachschule Hauswirtschaft der Berufsbildenden Schulen in Holzminden. Von 1978 bis 1981 absolvierte ich eine Krankenpflegeausbildung am evangelischen Krankenhaus Holzminden. Im Anschluss besuchte ich von 1981 bis 1984 das Fachgymnasium Hauswirtschaft der Berufsbildenden Schulen Holzminden. Im Juni 1984 erlangte ich die allgemeine Hochschulreife. Im folgenden Jahr war ich als Krankenschwester am evangelischen Krankenhaus Holzminden tätig. Im Jahr 1985 begann ich mit dem Studium der Psychologie an der Georg-August-Universität Göttingen. Das Studium der Humanmedizin wurde im Wintersemester 1989 an der Georg-August-Universität Göttingen begonnen. Den dritten Teil der ärztlichen Prüfung legte ich im Juni 1997 am Ev. Krankenhaus Weende ab. Nach meiner Tätigkeit als Ärztin im Praktikum am Institut für klinische Anästhesiologie und operative Intensivmedizin am Ev. Krankenhaus Göttingen Weende erhielt ich Dezember 1998 die Vollapprobation. Der Beginn meiner ärztlichen Tätigkeit erfolgte ab März 1999 als Assistenzärztin am Institut für klinische Anästhesiologie und operative Intensivmedizin am Ev. Krankenhaus Weende in Göttingen. In der Zeit von November 2001 bis Juni 2007 war ich als Assistenzärztin am Zentrum Anaesthesiologie-, Rettungs- und Intensivmedizin an der Georg-August-Universität Göttingen tätig. Die Facharztprüfung Anästhesie wurde von mir im August 2006 erfolgreich abgelegt. Ab Juli 2007 bis Januar 2010 arbeitete ich als Fachärztin für Anästhesie am Ev. Krankenhaus Weende in Göttingen. Für die Ausbildung im Bereich spezielle Schmerztherapie wechselte ich von Februar 2010 bis Oktober 2010 nochmals in die Schmerzambulanz des Zentrums Anaesthesiologie-, Rettungs- und Intensivmedizin an der Georg-August-Universität Göttingen. Ab November 2010 bin ich wieder als Fachärztin für Anästhesie am Ev. Krankenhaus Weende in Göttingen tätig. 